Supporting information for

\title{
A New Thiophene-Functionalized Pyrene, Peropyrene, and Teropyrene via a Two- or Four-fold Alkyne Annulation and Their Photophysical Properties
}

Wenlong Yang, Jorge H. S. K. Monteiro, Ana de Bettencourt-Dias and Wesley A. Chalifoux*

Department of Chemistry, University of Nevada, Reno, NV, 89557, USA Email: wchalifoux@unr.edu

\section{Table of contents}

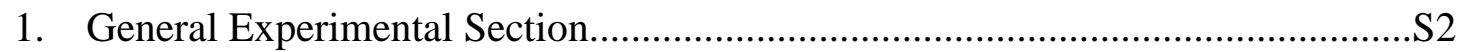

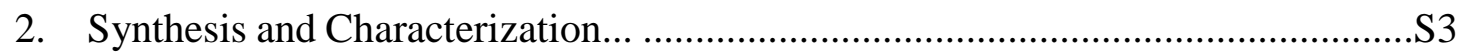

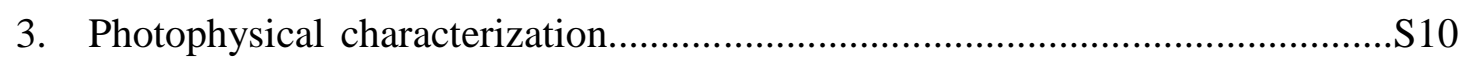

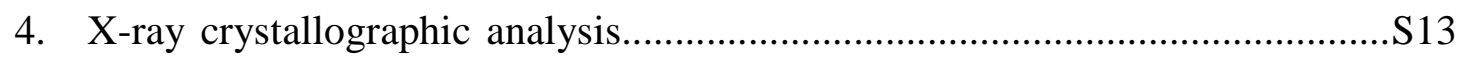

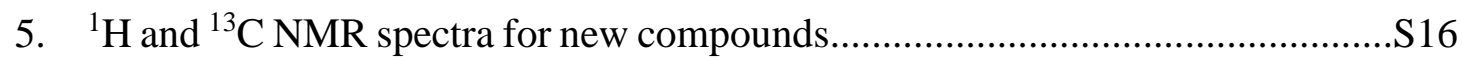

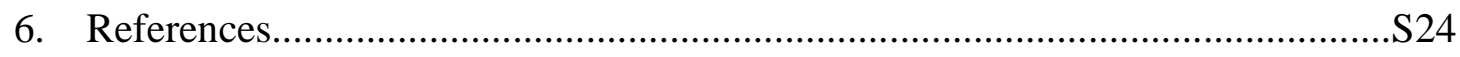




\section{General Experimental Section}

All reactions dealing with air- or moisture-sensitive compounds were carried out in a dry reaction vessel under nitrogen. Anhydrous tetrahydrofuran (THF) and dichloromethane $\left(\mathrm{CH}_{2} \mathrm{Cl}_{2}\right)$ were obtained by passing the solvent (HPLC grade) through an activated alumina column on a PureSolv MD 5 solvent drying system. ${ }^{1} \mathrm{H}$ and ${ }^{13} \mathrm{C}$ NMR spectra were recorded on Varian $400 \mathrm{MHz}$ or Varian $500 \mathrm{MHz}$ NMR Systems Spectrometers. Spectra were recorded in deuterated chloroform $\left(\mathrm{CDCl}_{3}\right)$. The residual protio-solvent peaks (7.26 ppm for ${ }^{1} \mathrm{H}$ and $77.16 \mathrm{ppm}$ for ${ }^{13} \mathrm{C}$, respectively) was used as an internal standard. Chemical shifts are reported in part per million (ppm) from low to high frequency and referenced to the residual solvent resonance. Coupling constants $(J)$ are reported in $\mathrm{Hz}$. The multiplicity of ${ }^{1} \mathrm{H}$ signals are indicated as: $\mathrm{s}=$ singlet, $\mathrm{d}=$ doublet, $\mathrm{t}=$ triplet, $\mathrm{m}=$ multiplet, $\mathrm{br}=$ broad. High resolution ESI mass spectrometry was recorded using an Agilent 6230 TOF MS and TFA was added to samples to promote ionization. MALDI-TOF mass spectra were recorded on a Bruker microflex MALDI-TOF spectrometer. TLC information was recorded on Silica gel 60 F254 glass plates. Purification of reaction products was carried out by flash chromatography using Silica Gel 60 (230-400 mesh).

A suitable crystal was mounted on a glass fiber and placed in the low-temperature nitrogen stream. Data were collected on a Bruker SMART CCD area detector diffractometer equipped with a low-temperature device, using graphitemonochromated Mo K $\alpha$ radiation $(\lambda=0.71073 \AA)$ and a full sphere of data was collected. Cell parameters were retrieved using SMART ${ }^{[1]}$ software and refined using SAINTPlus ${ }^{[2]}$ on all observed reflections. Data reduction and correction for Lp and decay were performed using the SAINTPlus ${ }^{[2]}$ software. Multi-scan absorption corrections were applied using SADABS, ${ }^{[3]}$ unless otherwise indicated. The structures were solved by direct methods and refined by least square methods on $\mathrm{F}^{2}$ using the SHELXTL ${ }^{[4]}$ program package. All non-hydrogen atoms were refined anisotropically. The majority of the hydrogen atoms were added geometrically and their parameters constrained to the parent site. 


\section{Synthesis and Characterization}

Compounds 1 was synthesized according to our previous report, ${ }^{[5]}$ and compound $\mathbf{2}$ was synthesized according to the literature. ${ }^{[6]}$

Note: For ${ }^{13} \mathrm{C}$ NMR spectra of compounds $\mathbf{4}$ and $\mathbf{6}$, one carbon signal was missed due to coincidental overlap.

\subsection{Synthesis of compound 4}

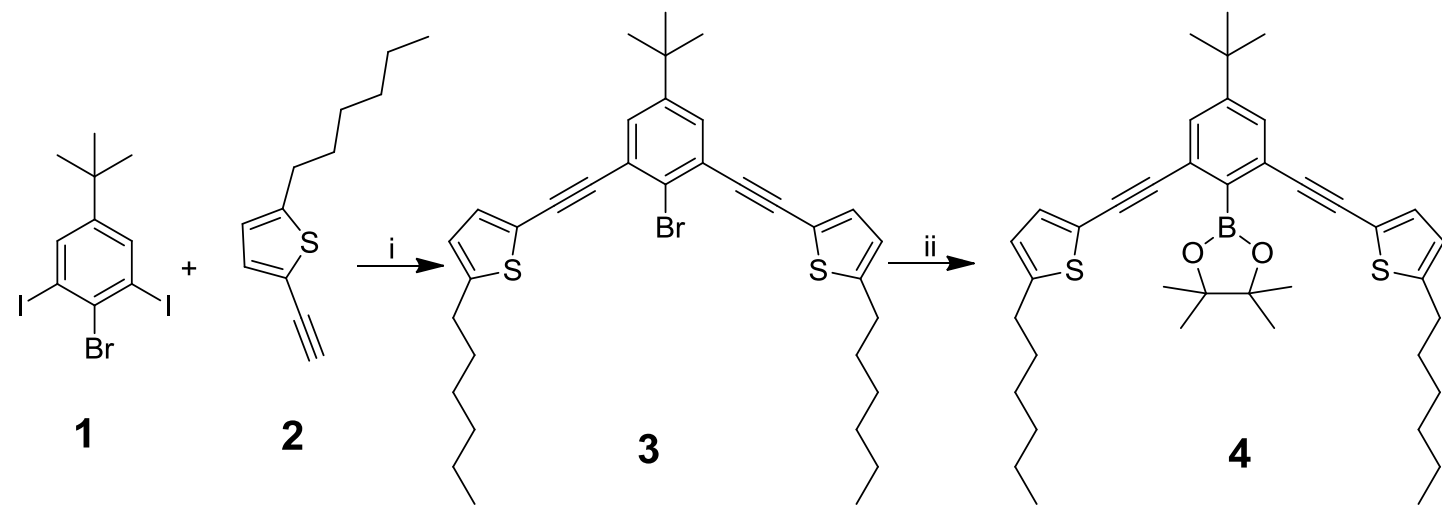

Scheme S1. Conditions: i) $\mathrm{Pd}\left(\mathrm{PPh}_{3}\right)_{2} \mathrm{Cl}_{2}, \mathrm{CuI}, \mathrm{THF} / \mathrm{Et}_{3} \mathrm{~N}$, r.t., $14 \mathrm{~h}$; ii) (a) $n$ butyllithium ( $n$-BuLi), THF, $-78{ }^{\circ} \mathrm{C}, 30 \mathrm{~min}$; (b) isopropoxyboronic acid pinacol ester, $-78{ }^{\circ} \mathrm{C}$ to r.t.

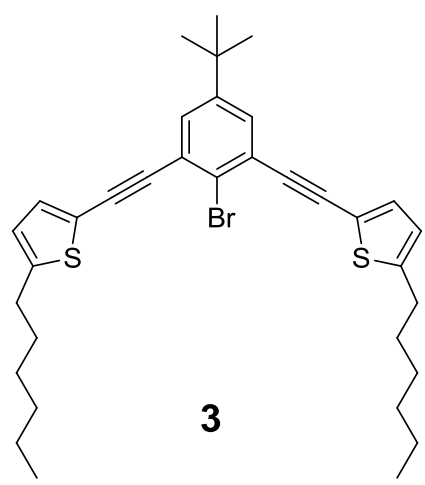

To the solution of 4-bromo-1-(tert-butyl)-3,5-diiodobenzene 1 (1.16 g, $2.50 \mathrm{mmol})$ and the terminal alkyne $2(1.15 \mathrm{~g}, 6.00 \mathrm{mmol})$ in $\mathrm{Et}_{3} \mathrm{~N}(20 \mathrm{~mL})$ and THF $(40 \mathrm{~mL})$, were added $\mathrm{Pd}\left(\mathrm{PPh}_{3}\right)_{2} \mathrm{Cl}_{2}(70.2 \mathrm{mg}, 0.100 \mathrm{mmol})$ and $\mathrm{CuI}(38.1 \mathrm{mg}, 0.200 \mathrm{mmol})$. The resulting mixture was stirred under a $\mathrm{N}_{2}$ atmosphere at room temperature for $14 \mathrm{~h}$. The ammonium salt was then removed by filtration. The solvent was removed under reduced pressure and the residue was purified by column chromatography (silica gel, hexane) to yield $\mathbf{3}$ as a yellow oil $(1.33 \mathrm{~g}, 90 \%)$. $\mathrm{R}_{f}=0.20$ (hexane).

FTIR (neat) 2954, 2925, 2854, 2204, 1565, $1465 \mathrm{~cm}^{-1}$. 
${ }^{1} \mathrm{H}$ NMR $\left(400 \mathrm{MHz}, \mathrm{CDCl}_{3}\right) \delta 7.50(\mathrm{~s}, 2 \mathrm{H}), 7.19(\mathrm{~d}, J=3.6 \mathrm{~Hz}, 2 \mathrm{H}), 6.71(\mathrm{~m}, 2 \mathrm{H}), 2.82$ (t, $J=7.6 \mathrm{~Hz}, 4 \mathrm{H}), 1.70(\mathrm{~m}, 4 \mathrm{H}), 1.54-1.17(\mathrm{~m}, 21 \mathrm{H}), 1.01-0.83(\mathrm{~m}, 6 \mathrm{H})$.

${ }^{13} \mathrm{C} \mathrm{NMR}\left(100 \mathrm{MHz}, \mathrm{CDCl}_{3}\right) \delta 150.19,149.22,132.65,129.85,125.89,124.60,124.46$, $120.09,91.59,87.46,34.66,31.66,31.64,31.09,30.39,28.84,22.70,14.21$.

HRMS (ESI, positive) $m / z$ calcd for $\mathrm{C}_{34} \mathrm{H}_{41} \mathrm{BrS}_{2}[\mathrm{M}+\mathrm{H}]^{+}$593.1911, found 593. 1902.

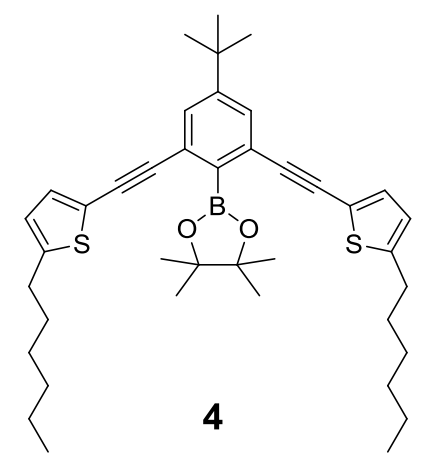

To a solution of $3(5.94 \mathrm{~g}, 10.0 \mathrm{mmol})$ in THF $(100 \mathrm{~mL})$ at $-78{ }^{\circ} \mathrm{C}$ was added a solution of $n$-butyllithium in hexanes $(4.00 \mathrm{~mL}, 2.5 \mathrm{M}, 10.0 \mathrm{mmol})$. After stirring for $30 \mathrm{~min}$ at $-78^{\circ} \mathrm{C}$, isopropoxyboronic acid pinacol ester $(1.86 \mathrm{~g}, 10.0 \mathrm{mmol})$ was added, the reaction removed from the cooling bath and allowed to warm. Upon reaching room temperature the reaction was quenched by the addition of $\mathrm{H}_{2} \mathrm{O}$, and then extracted with $\mathrm{CH}_{2} \mathrm{Cl}_{2}$. The extract was washed with water, dried with $\mathrm{Na}_{2} \mathrm{SO}_{4}$, filtered and concentrated in vacuo. The residue was purified by flash column chromatography (silica gel, hexane: $\mathrm{CH}_{2} \mathrm{Cl}_{2}=4: 1$, v/v) to yield 4 as light yellow oil $(4.70 \mathrm{~g}, 73 \%) . \mathrm{R}_{f}=$ 0.20 (hexane/ $\mathrm{CH}_{2} \mathrm{Cl}_{2} 4: 1$ ).

FTIR (neat) 2955, 2926, 2855, 2200, 1585, 1542, $1466 \mathrm{~cm}^{-1}$.

${ }^{1} \mathrm{H}$ NMR (400 MHz, $\left.\mathrm{CDCl}_{3}\right) \delta 7.49(\mathrm{~s}, 2 \mathrm{H}), 7.10(\mathrm{~d}, J=3.6 \mathrm{~Hz}, 2 \mathrm{H}), 6.67$ (d, $J=3.6$ $\mathrm{Hz}, 2 \mathrm{H}), 2.80$ (t, $J=7.5 \mathrm{~Hz}, 4 \mathrm{H}), 1.74-1.64(\mathrm{~m}, 4 \mathrm{H}), 1.57-1.17$ (m, 33H), 0.91 (t, $J$ $=6.9 \mathrm{~Hz}, 6 \mathrm{H})$.

${ }^{13} \mathrm{C}$ NMR $\left(100 \mathrm{MHz}, \mathrm{CDCl}_{3}\right) \delta 152.29,148.21,131.88,128.75,126.55,124.19,120.86$, 93.05, 84.36, 83.89, 34.71, 31.61, 31.55, 31.03, 30.28, 28.76, 25.14(2), 22.65, 14.15.

MALDI-TOFMS $m / z$ calcd for $\mathrm{C}_{40} \mathrm{H}_{53} \mathrm{BO}_{2} \mathrm{~S}_{2}[\mathrm{M}]^{+}$640.358, found 640.677 .

\subsection{Synthesis of compound 7}




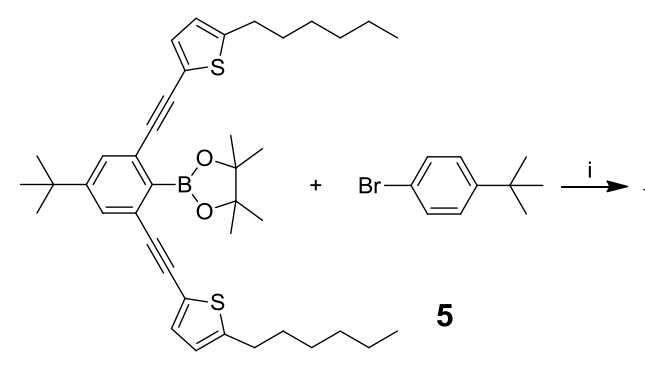

4

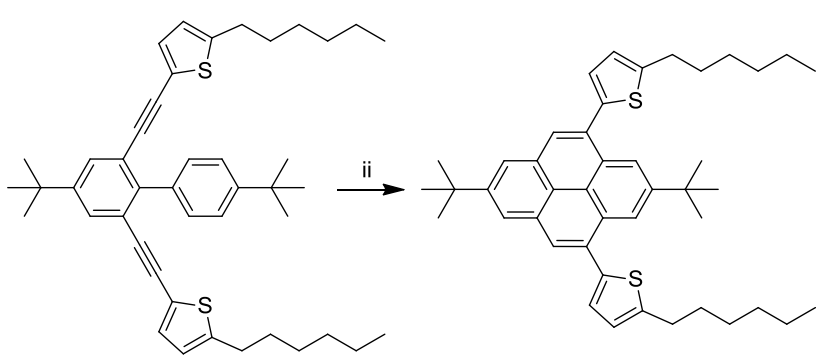

6

7

Scheme S2. Conditions. i) $\mathrm{Pd}\left(\mathrm{PPh}_{3}\right)_{4}, \mathrm{~K}_{2} \mathrm{CO}_{3}, \mathrm{THF} / \mathrm{H}_{2} \mathrm{O}, 70{ }^{\circ} \mathrm{C}, 12 \mathrm{~h}$; ii) $\mathrm{TfOH}, \mathrm{CH}_{2} \mathrm{Cl}_{2}$, $0{ }^{\circ} \mathrm{C}$.

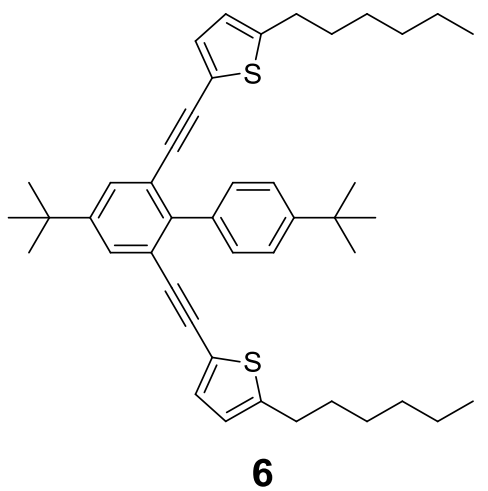

1-Bromo-4-tert-butylbenzene 5 (213 mg, $1.00 \mathrm{mmol})$, 2,6-diynylphenyl borate 4 (641 mg, $1.00 \mathrm{mmol})$ and $\mathrm{K}_{2} \mathrm{CO}_{3}(276 \mathrm{mg}, 2.00 \mathrm{mmol})$ were dissolved in THF (60 mL) and water $(10 \mathrm{~mL})$ solution. $\mathrm{Pd}\left(\mathrm{PPh}_{3}\right)_{4}(58.0 \mathrm{mg}, 0.0502 \mathrm{mmol})$ was added to the solution before degassing the mixture via bubbling nitrogen for $30 \mathrm{~min}$. The resulting mixture was stirred under a $\mathrm{N}_{2}$ atmosphere at $70{ }^{\circ} \mathrm{C}$ for $24 \mathrm{~h}$. After the reaction was complete, the mixture was diluted with $\mathrm{CH}_{2} \mathrm{Cl}_{2}$, washed with $\mathrm{H}_{2} \mathrm{O}$ and dried over $\mathrm{Na}_{2} \mathrm{SO}_{4}$. The solvent was removed under reduced pressure and the residue was purified by column chromatography (silica gel, hexane: $\mathrm{CH}_{2} \mathrm{Cl}_{2}=15: 1$, v/v) to yield $\mathbf{6}$ as yellow oil (375 mg, 58\%). $\mathrm{R}_{f}=0.30$ (hexane/ $\mathrm{CH}_{2} \mathrm{Cl}_{2}$ 10:1).

FTIR (neat) 2954, 2926, 2855, 2201, 1587, 1544, $1465 \mathrm{~cm}^{-1}$.

${ }^{1} \mathrm{H} \mathrm{NMR}\left(400 \mathrm{MHz}, \mathrm{CDCl}_{3}\right) \delta 7.59(\mathrm{~s}, 2 \mathrm{H}), 7.54(\mathrm{~m}, 4 \mathrm{H}), 6.86(\mathrm{~d}, J=3.6 \mathrm{~Hz}, 2 \mathrm{H}), 6.62$ $(\mathrm{d}, J=3.6 \mathrm{~Hz}, 2 \mathrm{H}), 2.77(\mathrm{t}, J=7.6 \mathrm{~Hz}, 4 \mathrm{H}), 1.71-1.59(\mathrm{~m}, 4 \mathrm{H}), 1.57-1.18(\mathrm{~m}, 30 \mathrm{H})$, $0.92(\mathrm{t}, J=6.8 \mathrm{~Hz}, 6 \mathrm{H})$.

${ }^{13} \mathrm{C} \mathrm{NMR}\left(100 \mathrm{MHz}, \mathrm{CDCl}_{3}\right) \delta 150.18,149.86,148.45,142.95,135.78,131.61,130.07$, $128.79,124.43,124.16,122.87,120.89$, 92.97, 86.26, 34.79, 34.67, 31.67, 31.62(2), $31.28,30.36,28.82,22.68,14.22$.

HRMS (ESI, positive) $m / z$ calcd for $\mathrm{C}_{44} \mathrm{H}_{54} \mathrm{~S}_{2}[\mathrm{M}+\mathrm{H}]^{+}$647.3740, found 647.3739. 


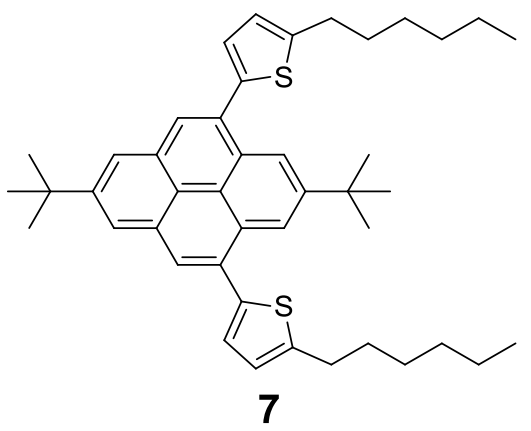

A $100 \mathrm{~mL}$ flame-dried flask was charged with compound 6 (35.0 $\mathrm{mg}, 0.0541 \mathrm{mmol})$ and anhydrous $\mathrm{CH}_{2} \mathrm{Cl}_{2}(50 \mathrm{~mL})$. A solution of triflic acid $(3.00 \mathrm{mg}, 0.0199 \mathrm{mmol})$ in anhydrous $\mathrm{CH}_{2} \mathrm{Cl}_{2}(3 \mathrm{~mL})$ was then added slowly by syringe in 30 minutes. After stirring for 12 hours at room temperature, the reaction was quenched with saturated $\mathrm{NaHCO}_{3}$ solution $(5 \mathrm{~mL})$. The solution was then washed with $\mathrm{H}_{2} \mathrm{O}(2 \times 30 \mathrm{~mL})$ and dried $\left(\mathrm{Na}_{2} \mathrm{SO}_{4}\right)$. After removal of the solvent under reduced pressure, the residue was purified by column chromatography (silica gel, hexane: $\mathrm{CH}_{2} \mathrm{Cl}_{2}=10: 1$, v/v) to yield 7 as yellow oil $(21.0 \mathrm{mg}, 60 \%)$. $\mathrm{R}_{f}=0.50$ (hexane $\left./ \mathrm{CH}_{2} \mathrm{Cl}_{2} 7: 1\right)$.

FTIR (neat) 2953, 2923, 2853, 1601, $1457 \mathrm{~cm}^{-1}$.

${ }^{1} \mathrm{H} \mathrm{NMR}\left(400 \mathrm{MHz}, \mathrm{CDCl}_{3}\right) \delta 8.79(\mathrm{~s}, 2 \mathrm{H}), 8.21(\mathrm{~s}, 2 \mathrm{H}), 8.17(\mathrm{~s}, 2 \mathrm{H}), 7.30(\mathrm{~d}, J=3.4$ $\mathrm{Hz}, 2 \mathrm{H}), 6.96$ (d, $J=3.4 \mathrm{~Hz}, 2 \mathrm{H}), 2.98$ (t, $J=7.6 \mathrm{~Hz}, 4 \mathrm{H}), 1.83$ (dd, $J=15.2,7.6 \mathrm{~Hz}$, $4 \mathrm{H}), 1.61(\mathrm{~s}, 9 \mathrm{H}), 1.54(\mathrm{~s}, 9 \mathrm{H}), 1.41(\mathrm{~m}, 12 \mathrm{H}), 0.97(\mathrm{t}, J=7.1 \mathrm{~Hz}, 6 \mathrm{H})$.

${ }^{13} \mathrm{C}$ NMR $\left(101 \mathrm{MHz}, \mathrm{CDCl}_{3}\right) \delta 149.27,148.44,146.35,140.02,132.58,130.33,130.12$, $128.97,127.06,124.50,123.82,122.51,122.12$, 121.42, 35.80, 35.35, 32.05, 32.01, $31.92,31.83,30.45,29.04,22.81,14.30$.

HRMS (ESI, positive) $\mathrm{m} / z$ calcd for $\mathrm{C}_{44} \mathrm{H}_{54} \mathrm{~S}_{2}[\mathrm{M}]^{+}$646.3661, found 646.3651 .

\subsection{Synthesis of compound 10}

$4+$

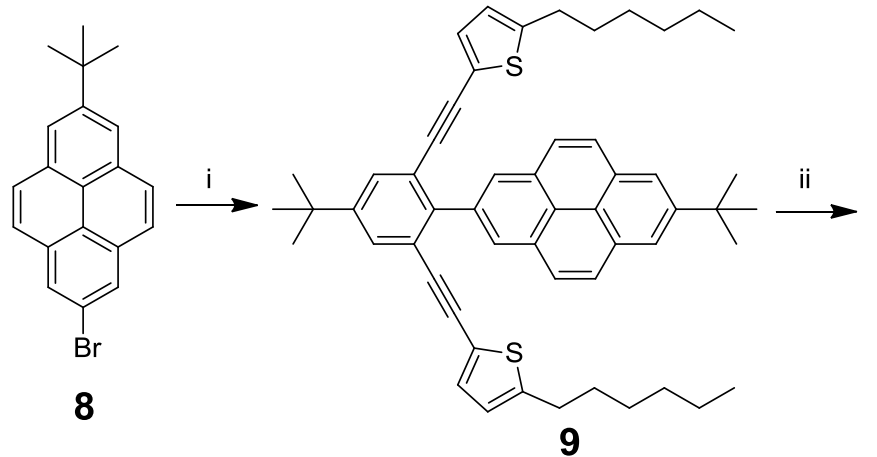

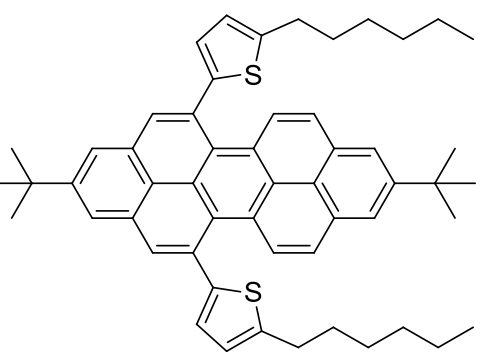

10

Scheme S3. Conditions. i) $\mathrm{Pd}\left(\mathrm{PPh}_{3}\right)_{4}, \mathrm{~K}_{2} \mathrm{CO}_{3}, \mathrm{THF} / \mathrm{H}_{2} \mathrm{O}, 70{ }^{\circ} \mathrm{C}, 24 \mathrm{~h}$; ii) $\mathrm{TfOH}, \mathrm{CH}_{2} \mathrm{Cl}_{2}$, $0{ }^{\circ} \mathrm{C}$. 


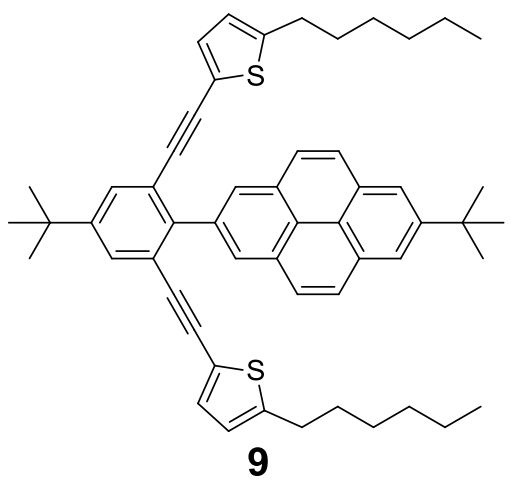

Compound 9 was prepared from 2,6-diynylphenyl borate 4 (320 mg, $0.499 \mathrm{mmol})$ and 2-bromo-7-(tert-butyl)pyrene $8(168 \mathrm{mg}, 0.498 \mathrm{mmol})$ according to the similar procedure to compound $\mathbf{6}$. The resulting residue was purified by column chromatography (silica gel, hexane: $\mathrm{CH}_{2} \mathrm{Cl}_{2}=15: 1$, v/v) to yield 9 as brown solid (169 $\mathrm{mg}, 49 \%$ ). $\mathrm{R}_{f}=0.30$ (hexane/ $\mathrm{CH}_{2} \mathrm{Cl}_{2}$ 10:1).

FTIR (neat) 2955, 2928, 2856, 2202, 1606, 1587, 1557, $1466 \mathrm{~cm}^{-1}$.

${ }^{1} \mathrm{H}$ NMR $\left(400 \mathrm{MHz}, \mathrm{CDCl}_{3}\right) \delta 8.51(\mathrm{~s}, 2 \mathrm{H}), 8.27(\mathrm{~s}, 2 \mathrm{H}), 8.13(\mathrm{~m}, 4 \mathrm{H}), 7.71(\mathrm{~s}, 2 \mathrm{H})$, $6.66(\mathrm{~d}, J=3.6 \mathrm{~Hz}, 2 \mathrm{H}), 6.46(\mathrm{~d}, J=3.6 \mathrm{~Hz}, 2 \mathrm{H}), 2.64(\mathrm{t}, J=7.6 \mathrm{~Hz}, 4 \mathrm{H}), 1.64(\mathrm{~s}, 9 \mathrm{H})$, $1.36(\mathrm{~d}, J=83.2 \mathrm{~Hz}, 34 \mathrm{H}), 0.88(\mathrm{t}, J=6.8 \mathrm{~Hz}, 6 \mathrm{H})$.

${ }^{13} \mathrm{C}$ NMR $\left(100 \mathrm{MHz}, \mathrm{CDCl}_{3}\right) \delta 150.37,149.04,148.49,142.51,135.87,131.87,131.42$, $130.37,129.34,127.97,127.41,127.35,124.20,124.15,123.33,123.16,122.08$, $120.41,92.87,86.35,35.40,34.79,32.14,31.61,31.48,31.32,30.23,28.74,22.65$, 14.19 .

HRMS (ESI, positive) $m / z$ calcd for $\mathrm{C}_{54} \mathrm{H}_{58} \mathrm{~S}_{2}[\mathrm{M}+\mathrm{H}]^{+}$771.4053, found 771.4048 .

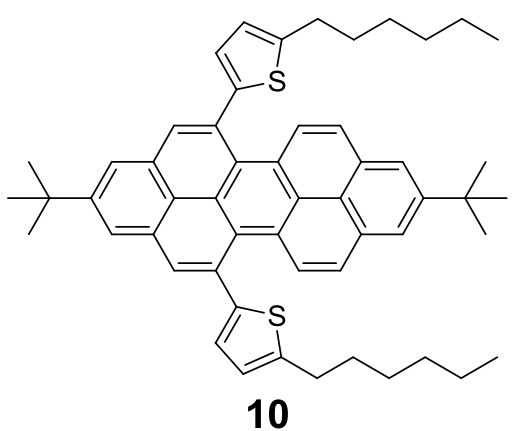

Compound 10 was prepared from compound $9(23.0 \mathrm{mg}, 0.0298 \mathrm{mmol})$ according to the similar procedure to compound 7 . The resulting residue was purified by column chromatography (silica gel, hexane: $\mathrm{CH}_{2} \mathrm{Cl}_{2}=10: 1$, v/v) to yield $\mathbf{1 0}$ as brown solid (16.6 $\mathrm{mg}, 72 \%$ ). $\mathrm{R}_{f}=0.50$ (hexane/ $\mathrm{CH}_{2} \mathrm{Cl}_{2} 10: 1$ ).

FTIR (neat) 2952, 2921, 2852, $1459 \mathrm{~cm}^{-1}$. 
${ }^{1} \mathrm{H} \mathrm{NMR}\left(400 \mathrm{MHz}, \mathrm{CDCl}_{3}\right) \delta 8.57(\mathrm{~d}, J=9.4 \mathrm{~Hz}, 2 \mathrm{H}), 8.43(\mathrm{~s}, 2 \mathrm{H}), 8.40(\mathrm{~s}, 2 \mathrm{H}), 8.32$ (s, 2H), $7.94(\mathrm{~d}, J=9.4 \mathrm{~Hz}, 2 \mathrm{H}), 7.06(\mathrm{~d}, J=3.4 \mathrm{~Hz}, 2 \mathrm{H}), 6.91(\mathrm{~d}, J=3.4 \mathrm{~Hz}, 2 \mathrm{H}), 2.98$ (t, $J=7.4 \mathrm{~Hz}, 4 \mathrm{H}), 1.83(\mathrm{~m}, 4 \mathrm{H}), 1.75-1.59(\mathrm{~m}, 18 \mathrm{H}), 1.57-1.40(\mathrm{~m}, 12 \mathrm{H}), 1.02$ (t, $J=6.9 \mathrm{~Hz}, 6 \mathrm{H})$.

${ }^{13} \mathrm{C}$ NMR $\left(100 \mathrm{MHz}, \mathrm{CDCl}_{3}\right) \delta 149.81,149.27,146.50,145.17,132.39,131.90,130.98$, $130.75,127.65,126.17,125.85,125.25,125.01,124.89,124.65,124.49,123.07$, $122.62,122.58,122.39,35.33,35.30,32.07,32.01,31.97,31.87,30.46,28.89,22.88$, 14.37 .

HRMS (ESI, positive) $m / z$ calcd for $\mathrm{C}_{54} \mathrm{H}_{58} \mathrm{~S}_{2}[\mathrm{M}]^{+} 770.3974$, found 770.3966 .

\subsection{Synthesis of compound 13}

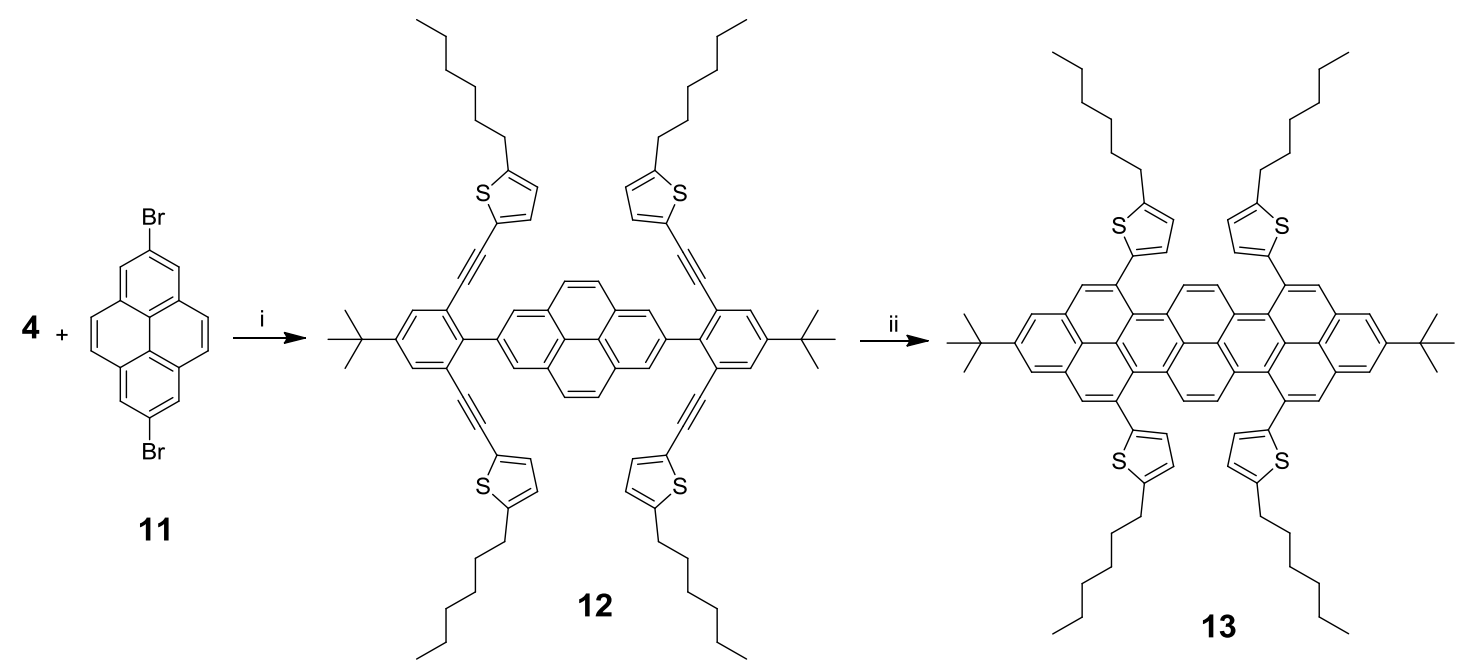

Scheme S3. Conditions. i) $\mathrm{Pd}\left(\mathrm{PPh}_{3}\right)_{4}, \mathrm{~K}_{2} \mathrm{CO}_{3}, \mathrm{THF} / \mathrm{H}_{2} \mathrm{O}, 70{ }^{\circ} \mathrm{C}, 48 \mathrm{~h}$; ii) $\mathrm{TfOH}, \mathrm{CH}_{2} \mathrm{Cl}_{2}$, $0{ }^{\circ} \mathrm{C}$ to r.t..

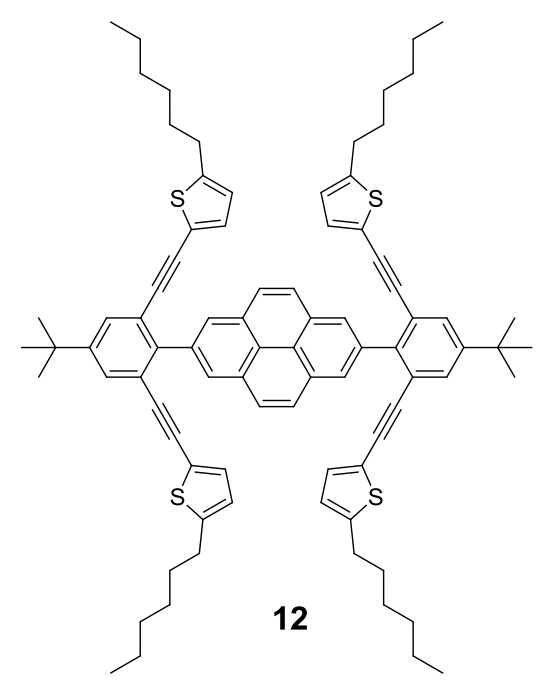

Compound 12 was prepared from 2,6-diynylphenyl borate 4 (641 mg, $1.00 \mathrm{mmol}$ ) 
and 2,7-dibromopyrene 11 (180 $\mathrm{mg}, 0.500 \mathrm{mmol})$ according to the similar procedure to compound 6 . The resulting residue was purified by column chromatography (silica gel, hexane: $\left.\mathrm{CH}_{2} \mathrm{Cl}_{2}=7: 1, \mathrm{v} / \mathrm{v}\right)$ to yield $\mathbf{1 2}$ as brown solid $(171 \mathrm{mg}, 28 \%) . \mathrm{R}_{f}=0.20$ (hexane $/ \mathrm{CH}_{2} \mathrm{Cl}_{2}$ 7:1).

FTIR (neat) 2953, 2924, 2853, 2199, 1587, 1554, $1464 \mathrm{~cm}^{-1}$.

${ }^{1} \mathrm{H}$ NMR $\left(400 \mathrm{MHz}, \mathrm{CDCl}_{3}\right) \delta 8.54(\mathrm{~s}, 4 \mathrm{H}), 8.18(\mathrm{~s}, 4 \mathrm{H}), 7.70(\mathrm{~s}, 4 \mathrm{H}), 6.67(\mathrm{~d}, J=3.6$ $\mathrm{Hz}, 4 \mathrm{H}), 6.43(\mathrm{~d}, J=3.6 \mathrm{~Hz}, 4 \mathrm{H}), 2.59$ (t, $J=7.5 \mathrm{~Hz}, 8 \mathrm{H}), 1.46(\mathrm{~s}, 18 \mathrm{H}), 1.24(\mathrm{~m}, 32 \mathrm{H})$, $0.87-0.80(\mathrm{~m}, 12 \mathrm{H})$.

${ }^{13} \mathrm{C} \mathrm{NMR}\left(100 \mathrm{MHz}, \mathrm{CDCl}_{3}\right) \delta 150.39,148.57,142.42,136.12,131.95,130.84,129.37$, $127.78,127.33,124.36,124.17,123.35,120.39,92.93,86.47,34.81,31.62,31.49$, $31.34,30.24,28.79,22.67,14.17$.

MALDI-TOFMS $m / z$ calcd for $\mathrm{C}_{84} \mathrm{H}_{90} \mathrm{~S}_{4}[\mathrm{M}+\mathrm{H}]^{+}$1227.600, found 1227.715.

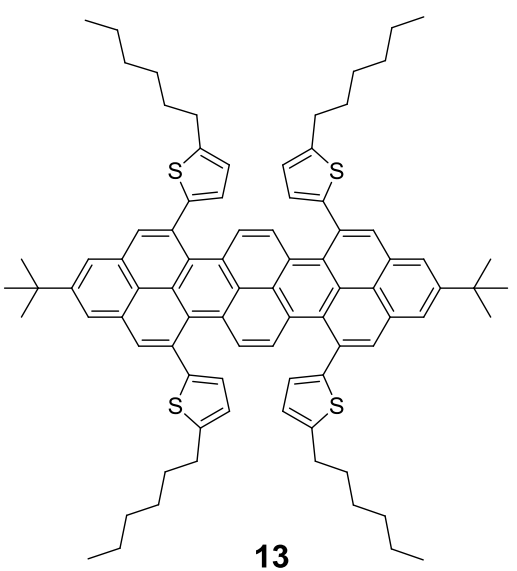

Compound 13 was prepared from compound $12(61.0 \mathrm{mg}, 0.0497 \mathrm{mmol})$ with triflic acid (2 equiv.) according to the similar procedure to compound 7. The resulting residue was purified by column chromatography (silica gel, hexane: $\mathrm{CH}_{2} \mathrm{Cl}_{2}=8: 1$, v/v) to yield 13 as brown solid (34.2 $\mathrm{mg}, 56 \%)$. $\mathrm{R}_{f}=0.20$ (hexane $/ \mathrm{CH}_{2} \mathrm{Cl}_{2}$ 8:1).

FTIR (neat) 2955, 2925, 2853, $1465 \mathrm{~cm}^{-1}$.

${ }^{1} \mathrm{H}$ NMR $\left(400 \mathrm{MHz}, \mathrm{CDCl}_{3}\right) \delta 8.35(\mathrm{~s}, 4 \mathrm{H}), 8.33(\mathrm{~s}, 4 \mathrm{H}), 8.31(\mathrm{~s}, 4 \mathrm{H}), 6.90(\mathrm{~d}, J=3.4$ $\mathrm{Hz}, 4 \mathrm{H}), 6.81(\mathrm{~d}, J=3.4 \mathrm{~Hz}, 4 \mathrm{H}), 2.94(\mathrm{t}, J=7.6 \mathrm{~Hz}, 8 \mathrm{H}), 1.81-1.75(\mathrm{~m}, 8 \mathrm{H}), 1.64-$ $1.61(\mathrm{~m}, 18 \mathrm{H}), 1.50-1.37(\mathrm{~m}, 24 \mathrm{H}), 0.91(\mathrm{~m}, 12 \mathrm{H})$.

${ }^{13} \mathrm{C} \mathrm{NMR}\left(100 \mathrm{MHz}, \mathrm{CDCl}_{3}\right) \delta 149.95,145.93,144.68,132.51,132.00,130.84,126.21$, 126.01, 125.50, 124.99, 124.87, 124.74, 124.45, 123.04, 122.09, 35.37, 32.01, 32.00, $31.83,30.59,29.06,22.82,14.30$.

MALDI-TOFMS $m / z$ calcd for $\mathrm{C}_{84} \mathrm{H}_{90} \mathrm{~S}_{4}[\mathrm{M}+\mathrm{H}]^{+} 1227.600$, found 1227.619. 


\section{Photophysical characterization}

Solutions with concentrations in the range $1 \times 10^{-5}$ to $1 \times 10^{-6} \mathrm{M}$ in toluene were used to obtain the absorption, emission and excitation spectra. The absorption spectra were measured on a Perkin Elmer Lambda 35 spectrometer. The photoluminescence data were obtained in a Fluorolog-3 spectrofluorimeter (Horiba FL3-22-iHR550), with 1200 grooves $/ \mathrm{mm}$ excitation monochromator gratings blazed at $330 \mathrm{~nm}$ and 1200 grooves $/ \mathrm{mm}$ emission monochromator gratings blazed at $500 \mathrm{~nm}$. An ozone-free xenon lamp of $450 \mathrm{~W}$ (Ushio) was used as the radiation source. The excitation spectra corrected for instrumental function were measured between 250 and $600 \mathrm{~nm}$. The emission spectra were measured in the range $350-800 \mathrm{~nm}$ at right angle. All emission spectra were corrected for instrumental function. Standards for quantum yield measurements were quinine sulfate $\left(\phi \sim 55 \%, 5 \times 10^{-6} \mathrm{M} \text { in aqueous } 0.5 \mathrm{M} \mathrm{H}_{2} \mathrm{SO}_{4}\right)^{[7,8]}$ for compounds pyrene 7 and peropyrene $\mathbf{1 0}$ and $\left[\mathrm{Ru}(\mathrm{bpy})_{3}\right] \mathrm{Cl}_{2}\left(\phi \sim 2.8 \%, 1 \times 10^{-5} \mathrm{M}\right.$ in water $)^{[8,9]}$ for compound teropyrene 13. Both samples and quantum yield standards were excited at the same wavelengths, which were chosen to ensure a linear relationship between the intensity of emitted light and the concentration of the absorbing/emitting species $(A \leq 0.05)$. The quantum yield of the samples was determined by the dilution method using Equation 1.

$$
\Phi_{x}=\frac{\operatorname{Grad}_{x}}{\operatorname{Grad}_{s t d}} \times \frac{n_{x}^{2}}{n_{\text {std }}^{2}} \times \Phi_{\text {std }}
$$

Grad is the slope of the plot 'Emission area vs Absorbance', $n$ is the refractive index of the solvent and $\Phi$ is the quantum yield for sample $x$ and standard std.

The emission decay curves were obtained using a TCSPC system and a Horiba NanoLED model N-370 (peak wavelength $=370 \pm 10 \mathrm{~nm}, \sim 4 \mathrm{pJ} /$ pulse) as excitation source. Before all decay curves measurements a blank, using Ludox ${ }^{\circledR}$ solution, was obtained.

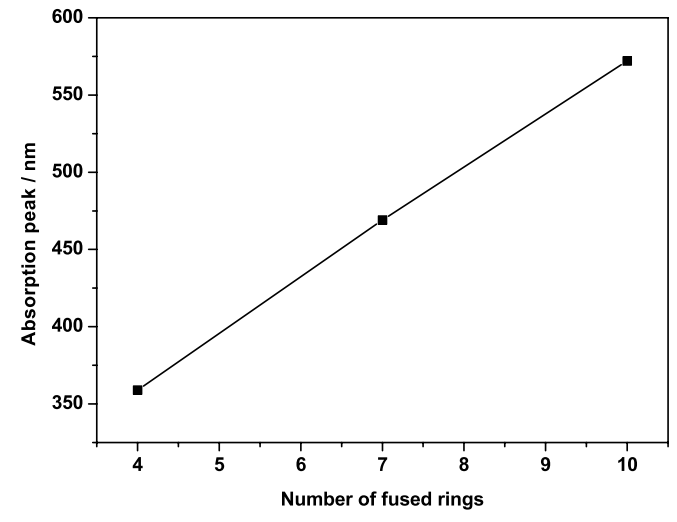

(a)

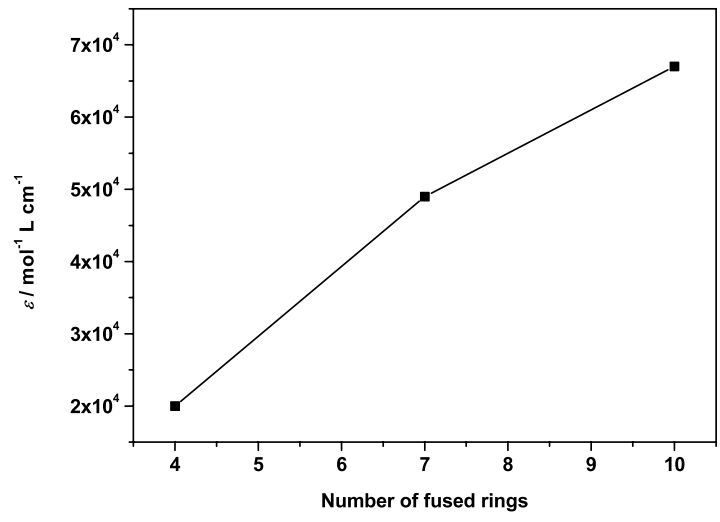

(b) 


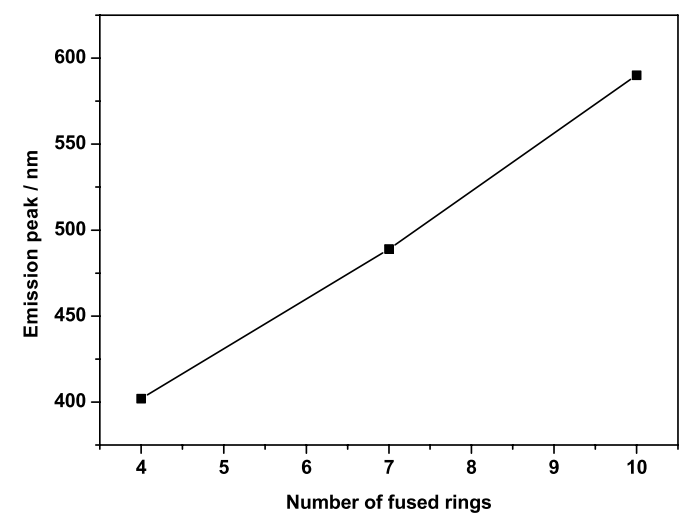

(c)

Figure S1. Correlation between (a) absorption peak, (b) molar absorptivity and (c) emission peak maximum and the number of fused rings.

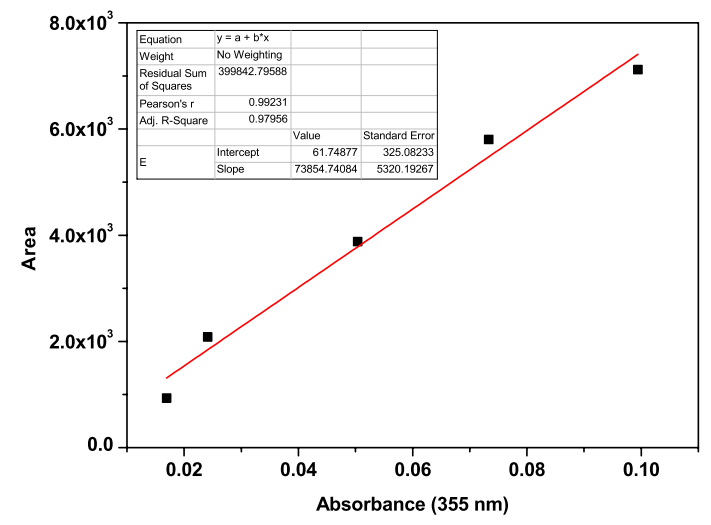

(a)

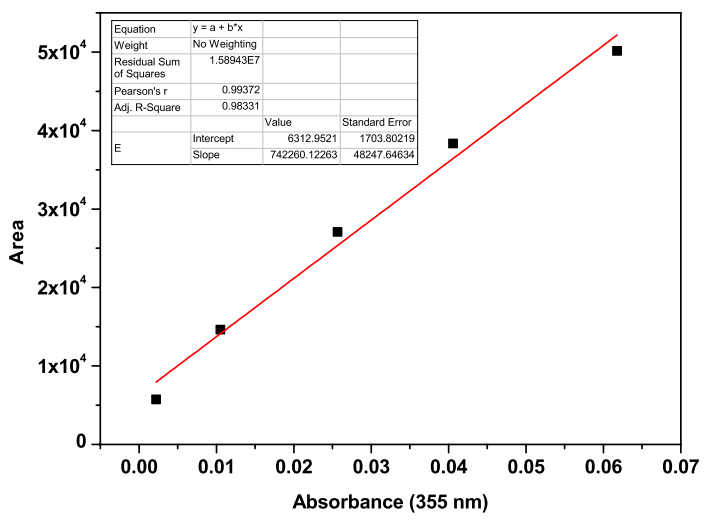

(b)

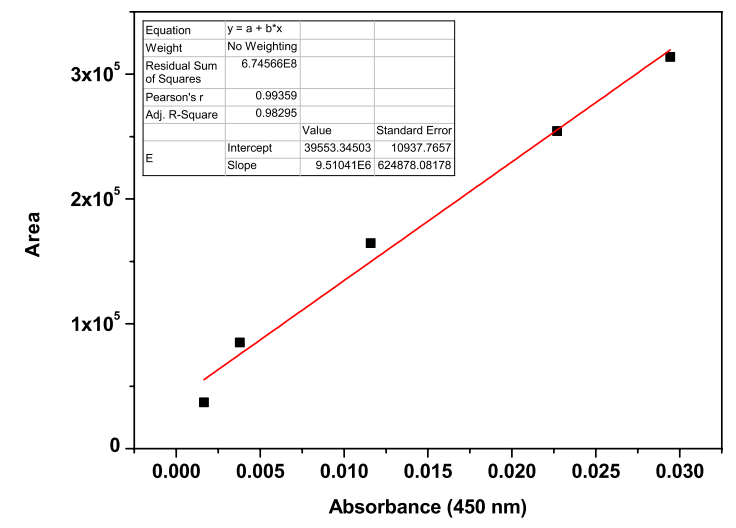

(c)

Figure S2. Plots of 'Emission area vs. Absorbance' for compounds (a) 7, (b) 10 and (c)

13. The concentrations of the solutions were in the range $1 \times 10^{-5}-1 \times 10^{-6} \mathrm{M}$ in toluene. Inserts show linear regression analysis of the fit (red lines). 


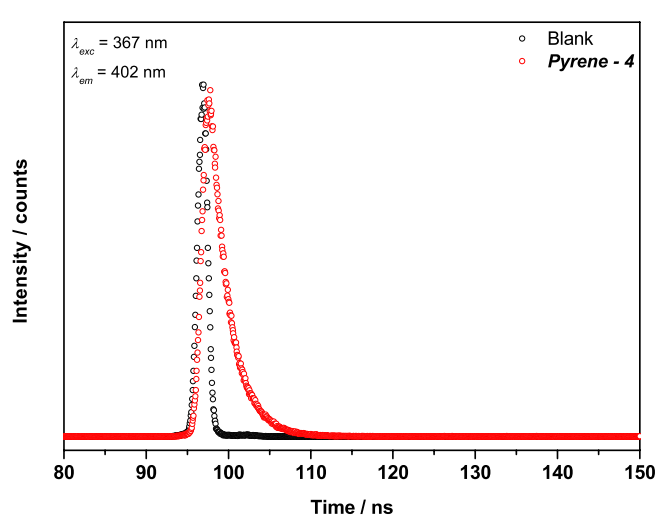

(a)

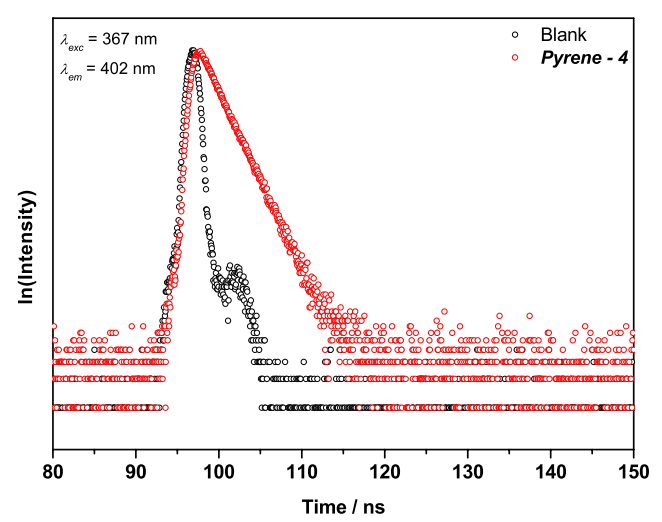

(b)

Figure S3. Emission decay curves for 7. (a) Exponential decay. (b) Linearization.

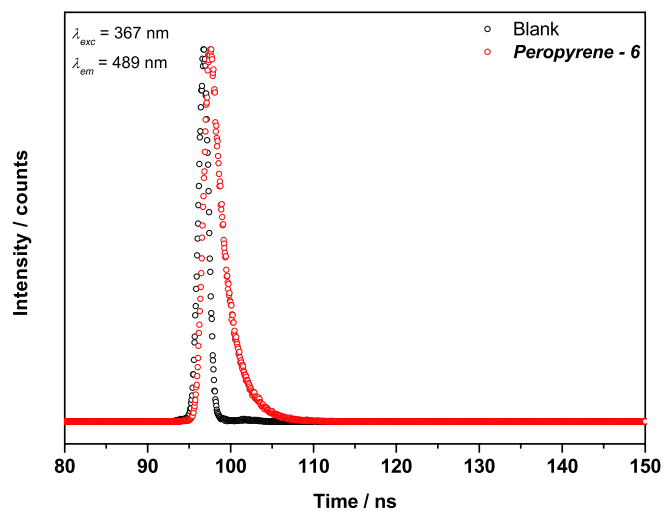

(a)

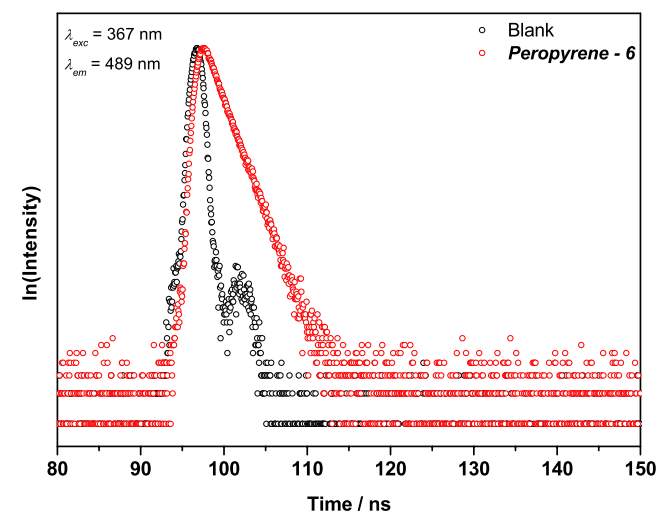

(b)

Figure S4. Emission decay curves for 10. (a) Exponential decay. (b) Linearization.

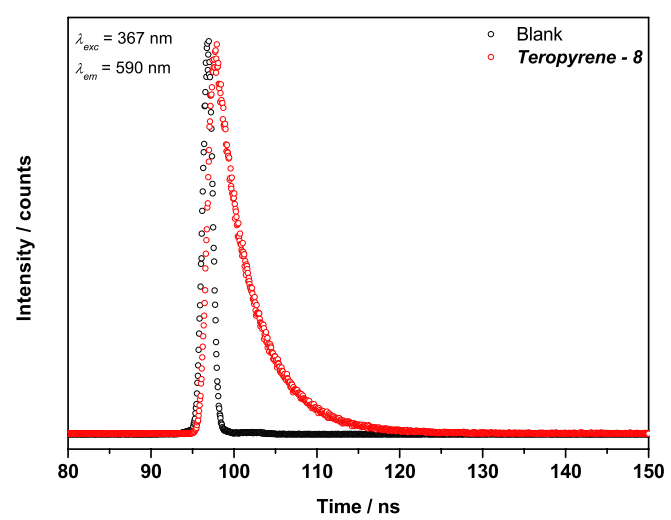

(a)

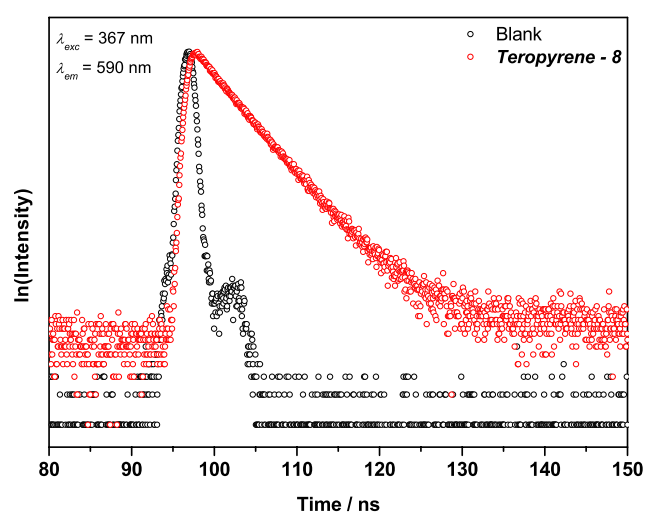

(b)

Figure S5. Emission decay curves for 13. (a) Exponential decay. (b) Linearization. 


\section{X-ray crystallographic analysis}

Crystallographic data for 10: $\mathrm{C}_{54} \mathrm{H}_{58} \mathrm{~S}_{2} ; M r=771.12$; crystal size $=0.715 \times 0.135 \times 0.100$ $\mathrm{mm}^{3}$; triclinic; space group $P-1 ; a=11.8559(5), b=13.4824(6), c=14.0263(6) \AA$; $\alpha=70.6239(8)^{\circ}, \beta=88.9436(8)^{\circ}, \gamma=87.6922(8)^{\circ} ; V=2113.31(16) \AA^{3} ; Z=2$, $\rho_{\text {calcd }}=1.212 \mathrm{Mg} / \mathrm{m}^{3} ; \mu=0.163 \mathrm{~mm}^{-1} ; \lambda=0.71073 \AA ̊ ㇒=100(2) \mathrm{K} ; 2 \theta_{\max }=60.00^{\circ}$; reflections measured 52815, independent $12321 \quad[R($ int $)=0.0432] ; \quad R_{l}=0.0646$, $w R_{2}=0.1831(\mathrm{I}>2 \sigma(\mathrm{I}))$; residual electron density $=1.417$ and $-0.617 \mathrm{e}^{-3}$. CCDC 1501742 (10) contains the supplementary crystallographic data for this paper. All these data can be obtained free of charge from The Cambridge Crystallographic Data Centre via www.ccdc.cam.ac.uk/data_request/cif.

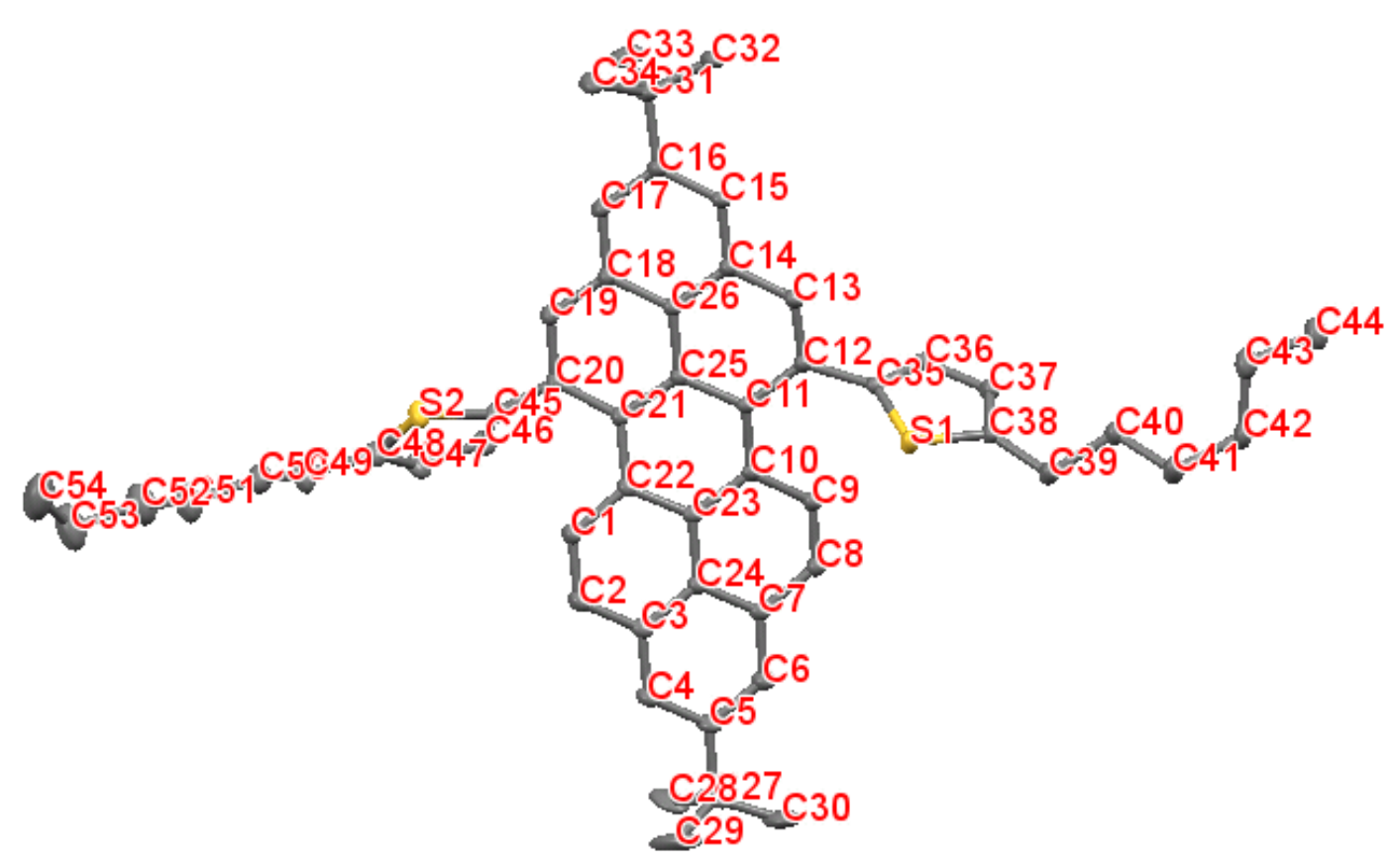

\subsection{Crystal data and structure refinement for 10}

Table S1 Crystal data and structure refinement for $\mathbf{1 0}$.

\begin{tabular}{lll}
\hline Empirical formula & C54 H58 S2 & \\
Formula weight & 771.12 & \\
Temperature & $100(2) \mathrm{K}$ & \\
Wavelength & $0.71073 \AA$ & \\
Crystal system & Triclinic & \\
Space group & $\mathrm{P}-1$ & $\mathrm{a}=70.6239(8)^{\circ}$. \\
Unit cell dimensions & $\mathrm{a}=11.8559(5) \AA$ & $\mathrm{b}=88.9436(8)^{\circ}$. \\
& $\mathrm{b}=13.4824(6) \AA$ & $\mathrm{g}=87.6922(8)^{\circ}$. \\
Volume & $\mathrm{c}=14.0263(6) \AA$ & \\
$\mathrm{Z}$ & $2113.31(16) \AA 3$ & \\
\hline
\end{tabular}




\begin{tabular}{ll}
\hline Density (calculated) & $1.212 \mathrm{Mg} / \mathrm{m}^{3}$ \\
Absorption coefficient & $0.163 \mathrm{~mm}^{-1}$ \\
$\mathrm{~F}(000)$ & 828 \\
Crystal size & $0.715 \times 0.135 \times 0.100 \mathrm{~mm}^{3}$ \\
Theta range for data collection & 1.539 to $29.999^{\circ}$. \\
Index ranges & $-16<=\mathrm{h}<=16,-18<=\mathrm{k}<=18,-19<=\mathrm{l}<=19$ \\
Reflections collected & 52815 \\
Independent reflections & $12321[\mathrm{R}(\mathrm{int})=0.0432]$ \\
Completeness to theta $=25.242^{\circ}$ & $99.9 \%$ \\
Absorption correction & Semi-empirical from equivalents \\
Max. and min. transmission & 0.7461 and 0.7140 \\
Refinement method & Full-matrix least-squares on $\mathrm{F}^{2}$ \\
Data / restraints / parameters & $12321 / 30 / 535$ \\
Goodness-of-fit on $\mathrm{F}^{2}$ & 1.113 \\
Final R indices [I $>2$ sigma(I)] & $\mathrm{R} 1=0.0646, \mathrm{wR} 2=0.1831$ \\
$\mathrm{R}$ indices (all data) & $\mathrm{R} 1=0.0869, \mathrm{wR} 2=0.1962$ \\
Extinction coefficient & $\mathrm{n} / \mathrm{a}$ \\
Largest diff. peak and hole & 1.417 and -0.617 e. $\AA^{-3}$ \\
\hline
\end{tabular}

Table S2 Selected bond lengths $[\AA]$ for $\mathbf{1 0}$.

\begin{tabular}{ll|ll|ll}
\hline $\mathrm{C}(1)-\mathrm{C}(2)$ & $1.355(3)$ & $\mathrm{C}(5)-\mathrm{C}(6)$ & $1.396(3)$ & $\mathrm{C}(9)-\mathrm{C}(10)$ & $1.447(2)$ \\
$\mathrm{C}(1)-\mathrm{C}(22)$ & $1.442(2)$ & $\mathrm{C}(5)-\mathrm{C}(27)$ & $1.531(3)$ & $\mathrm{C}(10)-\mathrm{C}(23)$ & $1.427(2)$ \\
$\mathrm{C}(2)-\mathrm{C}(3)$ & $1.428(3)$ & $\mathrm{C}(6)-\mathrm{C}(7)$ & $1.404(2)$ & $\mathrm{C}(10)-\mathrm{C}(11)$ & $1.429(2)$ \\
$\mathrm{C}(3)-\mathrm{C}(4)$ & $1.400(3)$ & $\mathrm{C}(7)-\mathrm{C}(24)$ & $1.412(2)$ & $\mathrm{C}(11)-\mathrm{C}(25)$ & $1.436(2)$ \\
$\mathrm{C}(3)-\mathrm{C}(24)$ & $1.420(2)$ & $\mathrm{C}(7)-\mathrm{C}(8)$ & $1.431(2)$ & $\mathrm{C}(11)-\mathrm{C}(12)$ & $1.454(2)$ \\
$\mathrm{C}(4)-\mathrm{C}(5)$ & $1.393(3)$ & $\mathrm{C}(8)-\mathrm{C}(9)$ & $1.357(2)$ & & \\
\hline
\end{tabular}

Table S3 Selected bond angles [ $\left.{ }^{\circ}\right]$ for $\mathbf{1 0}$.

\begin{tabular}{ll|ll}
\hline $\mathrm{C}(2)-\mathrm{C}(1)-\mathrm{C}(22)$ & $121.84(17)$ & $\mathrm{C}(24)-\mathrm{C}(7)-\mathrm{C}(8)$ & $118.08(16)$ \\
$\mathrm{C}(1)-\mathrm{C}(2)-\mathrm{C}(3)$ & $121.18(17)$ & $\mathrm{C}(9)-\mathrm{C}(8)-\mathrm{C}(7)$ & $121.34(17)$ \\
$\mathrm{C}(4)-\mathrm{C}(3)-\mathrm{C}(24)$ & $119.70(17)$ & $\mathrm{C}(8)-\mathrm{C}(9)-\mathrm{C}(10)$ & $122.19(17)$ \\
$\mathrm{C}(4)-\mathrm{C}(3)-\mathrm{C}(2)$ & $121.90(17)$ & $\mathrm{C}(23)-\mathrm{C}(10)-\mathrm{C}(11)$ & $118.92(15)$ \\
$\mathrm{C}(24)-\mathrm{C}(3)-\mathrm{C}(2)$ & $118.39(16)$ & $\mathrm{C}(23)-\mathrm{C}(10)-\mathrm{C}(9)$ & $116.97(15)$ \\
$\mathrm{C}(5)-\mathrm{C}(4)-\mathrm{C}(3)$ & $122.28(18)$ & $\mathrm{C}(11)-\mathrm{C}(10)-\mathrm{C}(9)$ & $124.06(16)$ \\
$\mathrm{C}(4)-\mathrm{C}(5)-\mathrm{C}(6)$ & $117.56(17)$ & $\mathrm{C}(10)-\mathrm{C}(11)-\mathrm{C}(25)$ & $117.99(15)$ \\
$\mathrm{C}(5)-\mathrm{C}(6)-\mathrm{C}(7)$ & $122.13(18)$ & $\mathrm{C}(10)-\mathrm{C}(11)-\mathrm{C}(12)$ & $124.29(16)$ \\
$\mathrm{C}(6)-\mathrm{C}(7)-\mathrm{C}(24)$ & $119.76(17)$ & $\mathrm{C}(25)-\mathrm{C}(11)-\mathrm{C}(12)$ & $117.70(15)$ \\
$\mathrm{C}(6)-\mathrm{C}(7)-\mathrm{C}(8)$ & $122.15(17)$ & & \\
\hline
\end{tabular}

Table S4 Selected torsion angles $\left[{ }^{\circ}\right]$ for $\mathbf{1 0}$.

\begin{tabular}{lr|lr}
\hline $\mathrm{C}(22)-\mathrm{C}(1)-\mathrm{C}(2)-\mathrm{C}(3)$ & $-1.5(3)$ & $\mathrm{C}(6)-\mathrm{C}(7)-\mathrm{C}(8)-\mathrm{C}(9)$ & $-176.25(18)$ \\
$\mathrm{C}(1)-\mathrm{C}(2)-\mathrm{C}(3)-\mathrm{C}(4)$ & $-175.81(19)$ & $\mathrm{C}(24)-\mathrm{C}(7)-\mathrm{C}(8)-\mathrm{C}(9)$ & $4.9(3)$ \\
\hline
\end{tabular}




\begin{tabular}{lr|lr}
\hline $\mathrm{C}(1)-\mathrm{C}(2)-\mathrm{C}(3)-\mathrm{C}(24)$ & $5.2(3)$ & $\mathrm{C}(7)-\mathrm{C}(8)-\mathrm{C}(9)-\mathrm{C}(10)$ & $-2.2(3)$ \\
$\mathrm{C}(24)-\mathrm{C}(3)-\mathrm{C}(4)-\mathrm{C}(5)$ & $-1.9(3)$ & $\mathrm{C}(8)-\mathrm{C}(9)-\mathrm{C}(10)-\mathrm{C}(23)$ & $-5.5(3)$ \\
$\mathrm{C}(2)-\mathrm{C}(3)-\mathrm{C}(4)-\mathrm{C}(5)$ & $179.10(19)$ & $\mathrm{C}(8)-\mathrm{C}(9)-\mathrm{C}(10)-\mathrm{C}(11)$ & $176.81(17)$ \\
$\mathrm{C}(3)-\mathrm{C}(4)-\mathrm{C}(5)-\mathrm{C}(6)$ & $0.2(3)$ & $\mathrm{C}(23)-\mathrm{C}(10)-\mathrm{C}(11)-\mathrm{C}(25)$ & $-16.0(2)$ \\
$\mathrm{C}(4)-\mathrm{C}(5)-\mathrm{C}(6)-\mathrm{C}(7)$ & $1.8(3)$ & $\mathrm{C}(9)-\mathrm{C}(10)-\mathrm{C}(11)-\mathrm{C}(25)$ & $161.61(17)$ \\
$\mathrm{C}(27)-\mathrm{C}(5)-\mathrm{C}(6)-\mathrm{C}(7)$ & $-179.2(2)$ & $\mathrm{C}(11)-\mathrm{C}(12)-\mathrm{C}(35)-\mathrm{S}(1)$ & $-72.2(2)$ \\
$\mathrm{C}(5)-\mathrm{C}(6)-\mathrm{C}(7)-\mathrm{C}(24)$ & $-2.2(3)$ & $\mathrm{C}(10)-\mathrm{C}(11)-\mathrm{C}(12)-\mathrm{C}(35)$ & $-3.1(3)$ \\
$\mathrm{C}(5)-\mathrm{C}(6)-\mathrm{C}(7)-\mathrm{C}(8)$ & $178.99(19)$ & & \\
\hline
\end{tabular}




\section{5. ${ }^{1} \mathrm{H}$ and ${ }^{13} \mathrm{C}$ NMR spectra for new compounds}

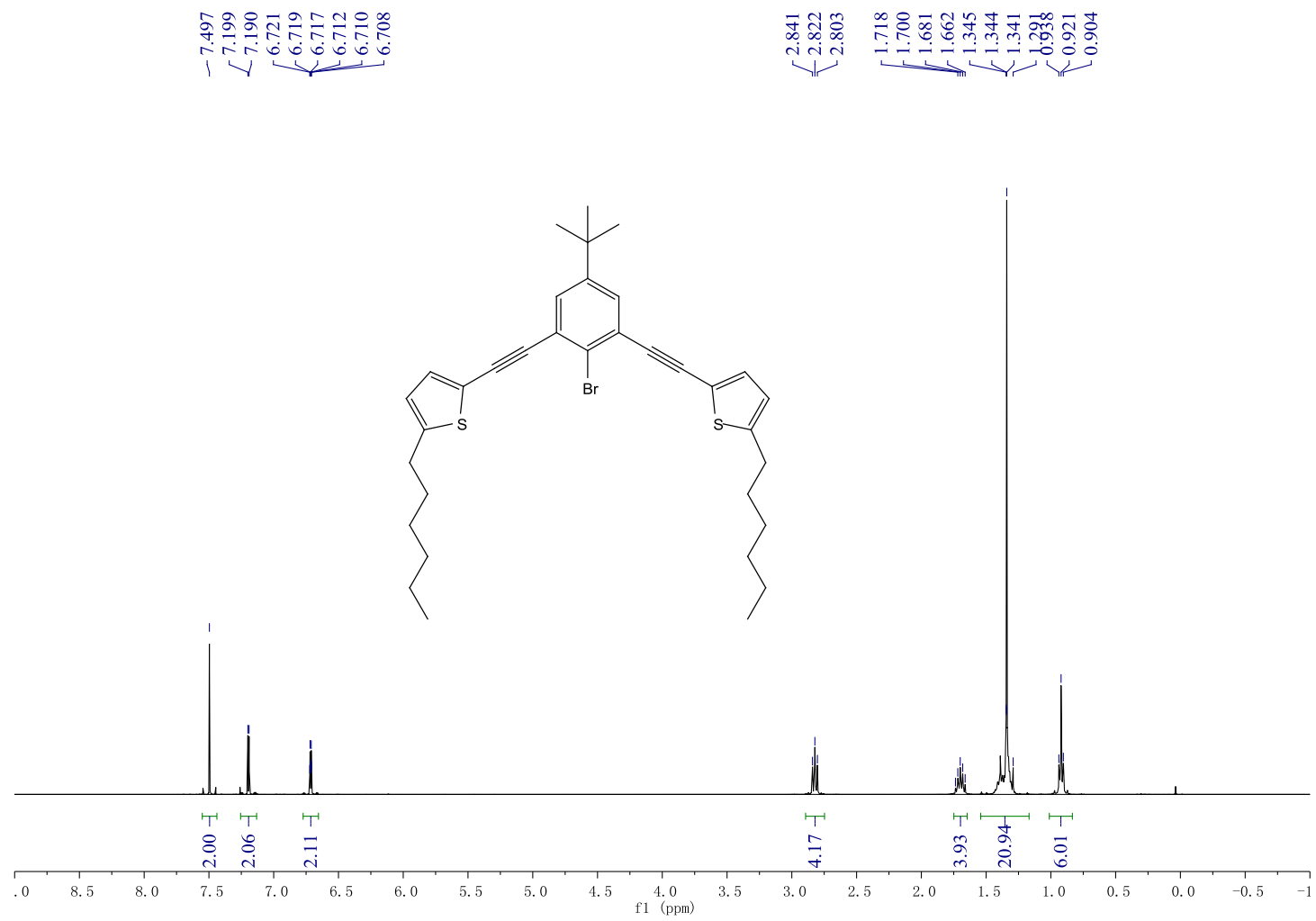

Figure S6. ${ }^{1} \mathrm{H}$ NMR spectrum for compound 3 in $\mathrm{CDCl}_{3}$ at $298 \mathrm{~K}$.

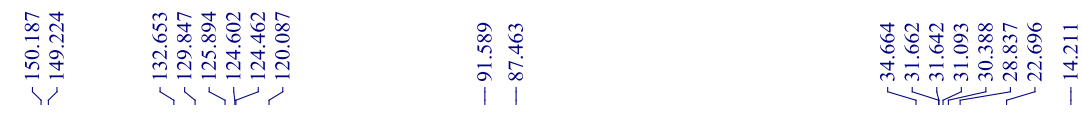

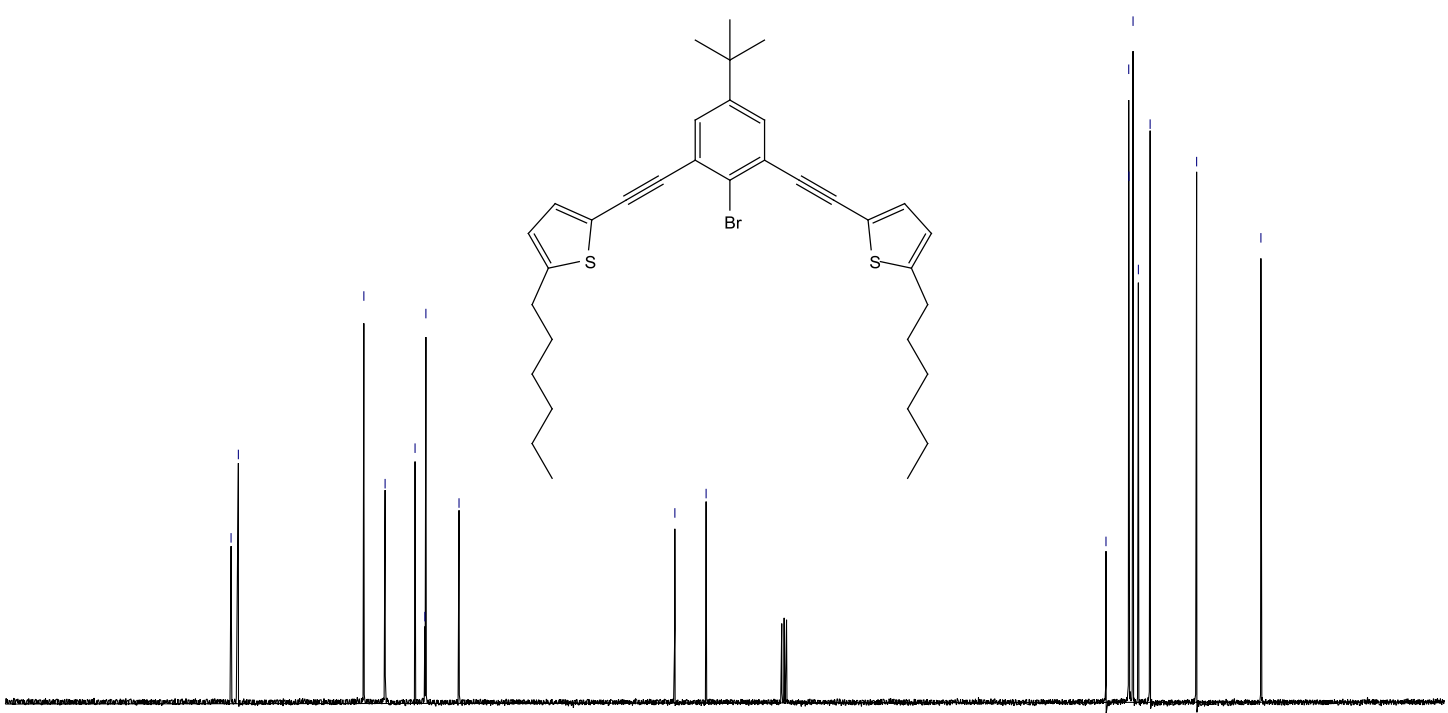

Figure S7. ${ }^{13} \mathrm{C}$ NMR spectrum for compound $\mathbf{3}$ in $\mathrm{CDCl}_{3}$ at $298 \mathrm{~K}$. 


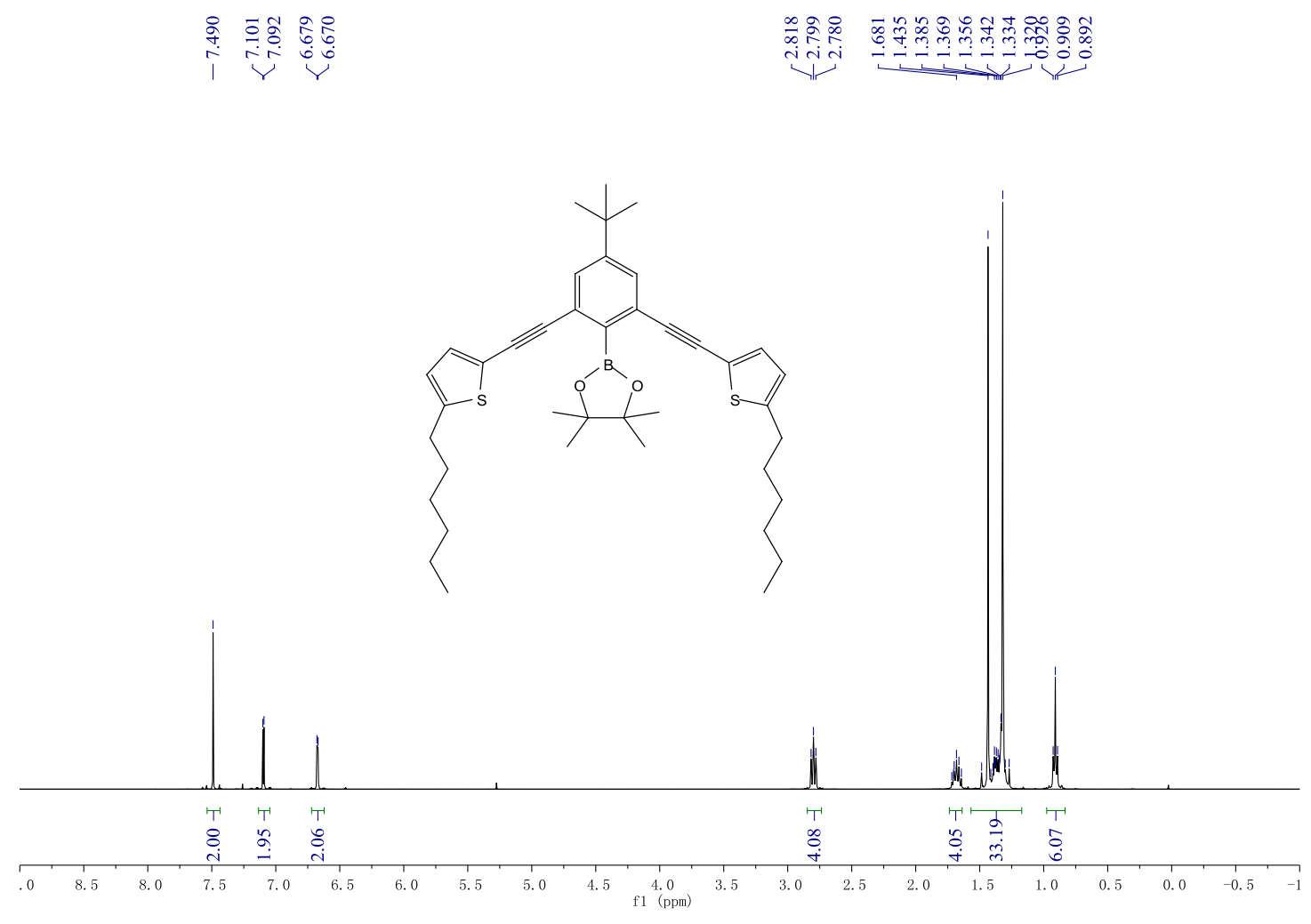

Figure S8. ${ }^{1} \mathrm{H}$ NMR spectrum for compound 4 in $\mathrm{CDCl}_{3}$ at $298 \mathrm{~K}$.
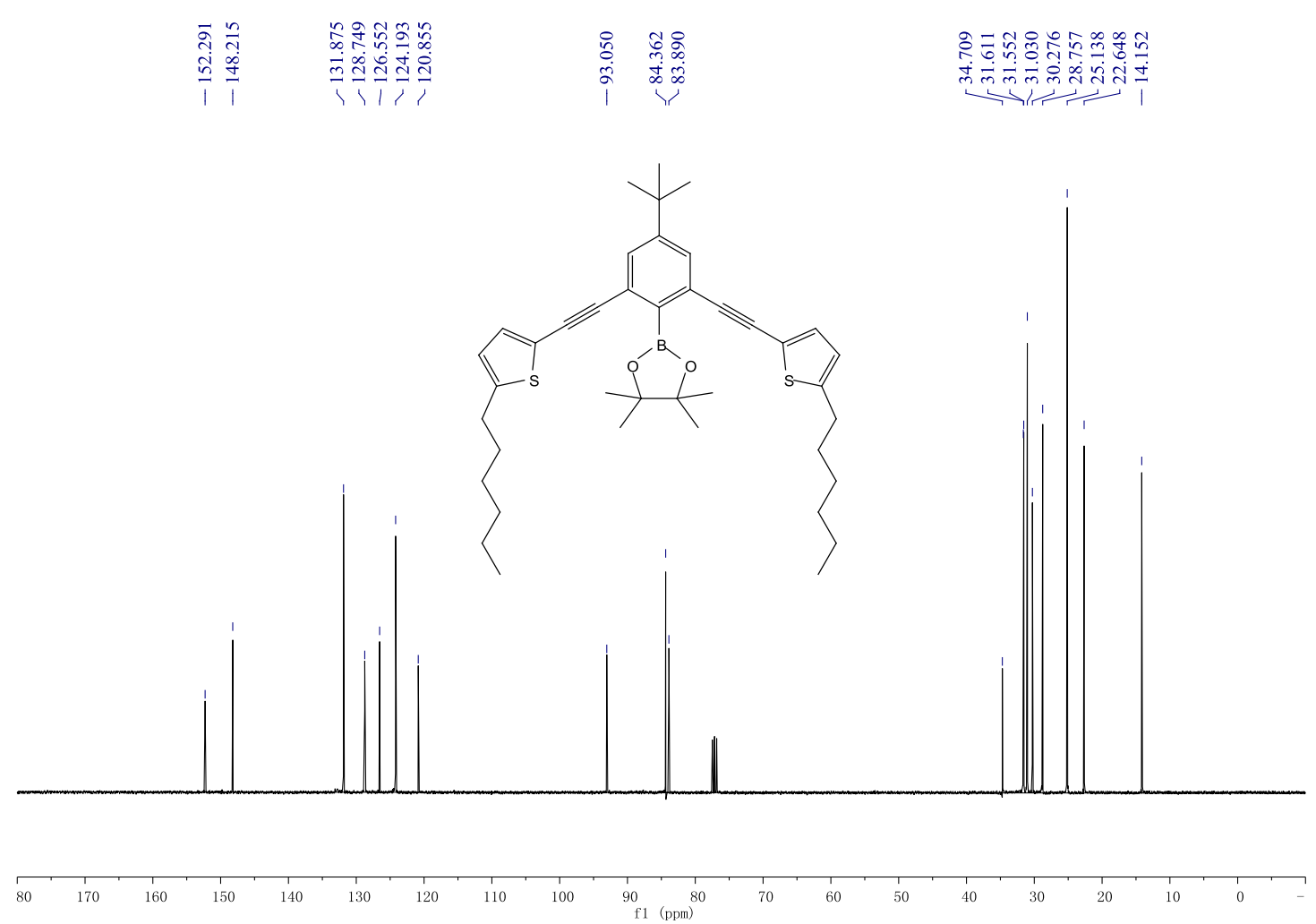

Figure S9. ${ }^{13} \mathrm{C}$ NMR spectrum for compound 4 in $\mathrm{CDCl}_{3}$ at $298 \mathrm{~K}$. 


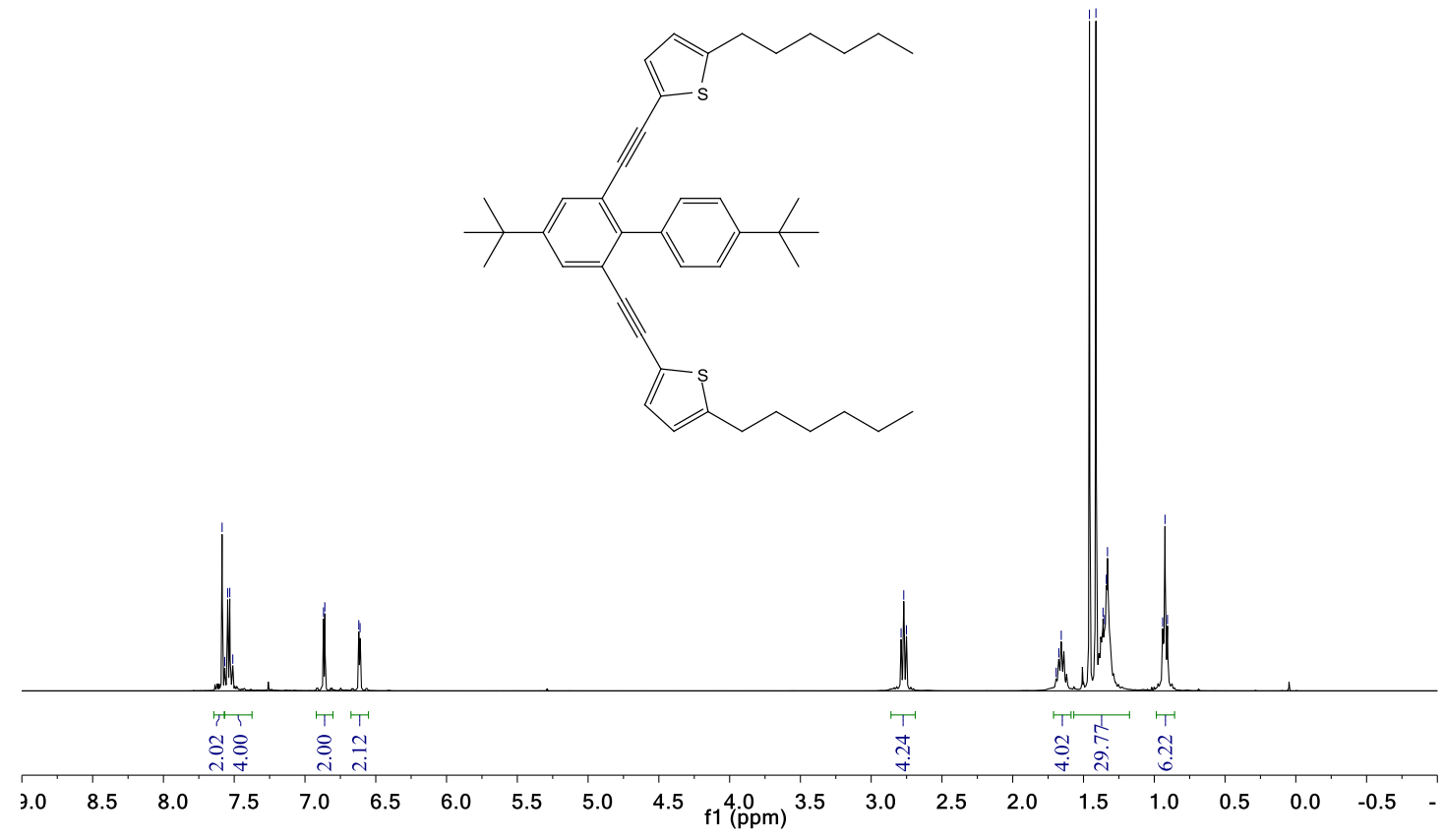

Figure S10. ${ }^{1} \mathrm{H}$ NMR spectrum for compound 6 in $\mathrm{CDCl}_{3}$ at $298 \mathrm{~K}$.
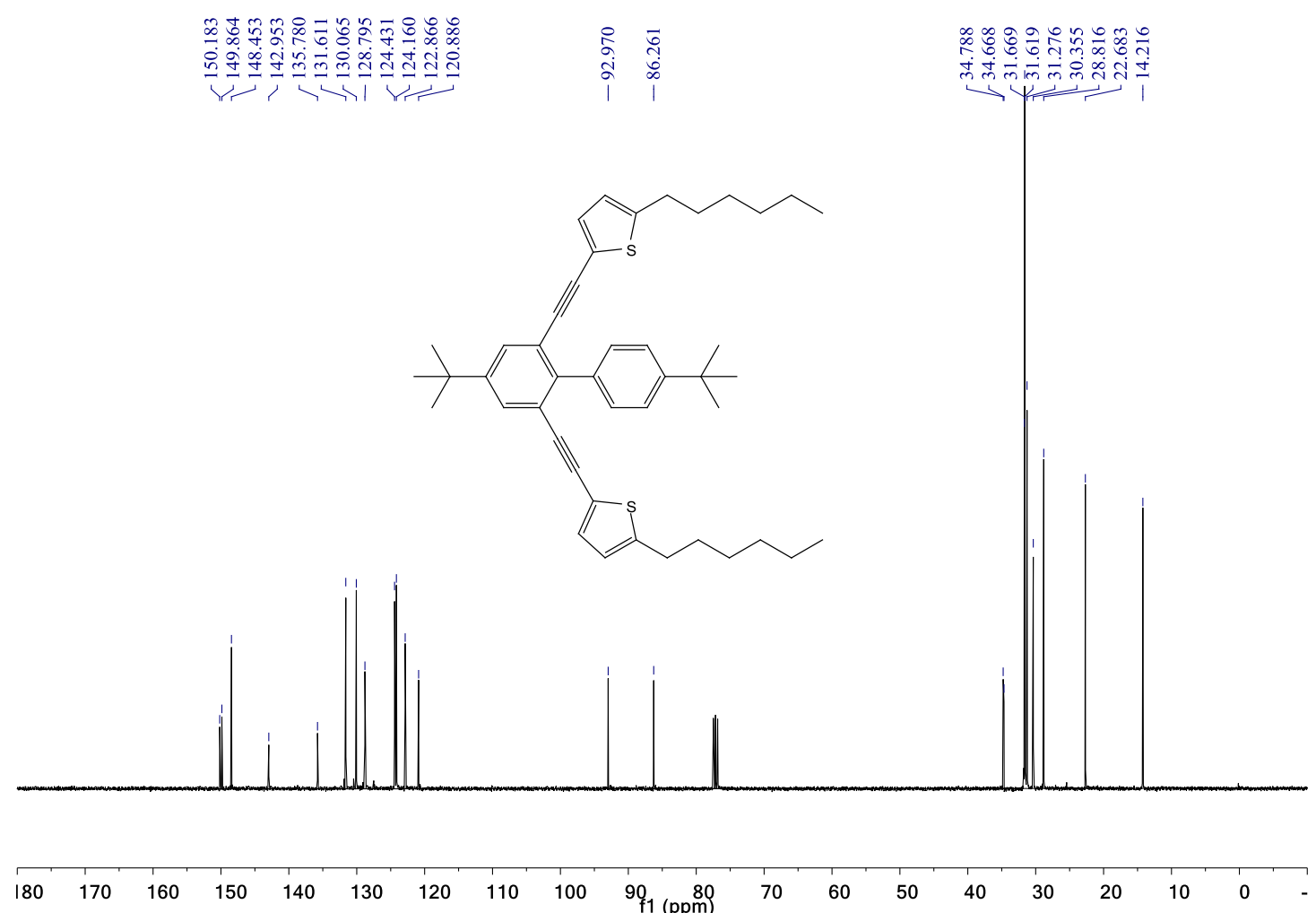

Figure S11. ${ }^{13} \mathrm{C}$ NMR spectrum for compound 6 in $\mathrm{CDCl}_{3}$ at $298 \mathrm{~K}$. 


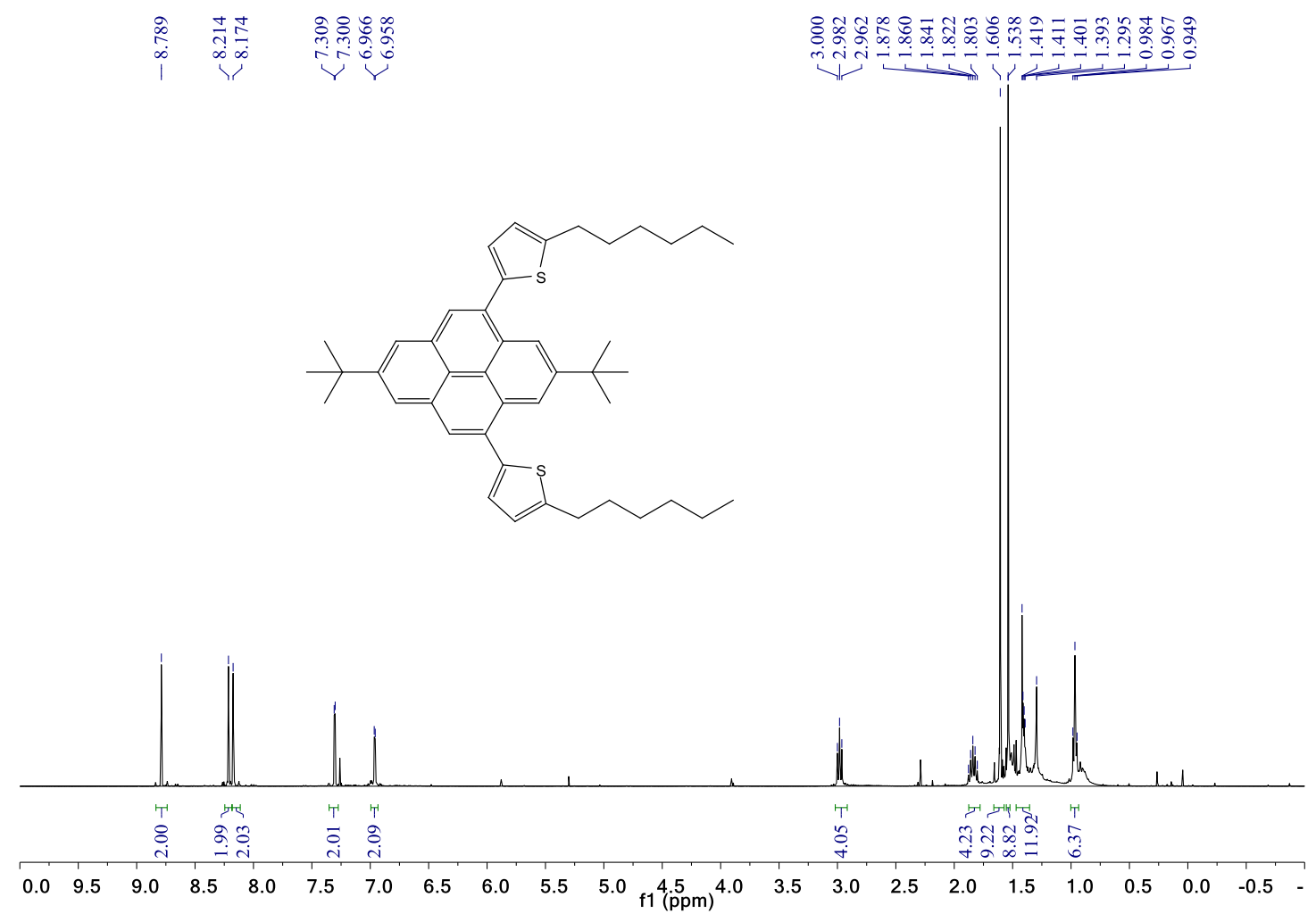

Figure S12. ${ }^{1} \mathrm{H}$ NMR spectrum for compound 7 in $\mathrm{CDCl}_{3}$ at $298 \mathrm{~K}$.
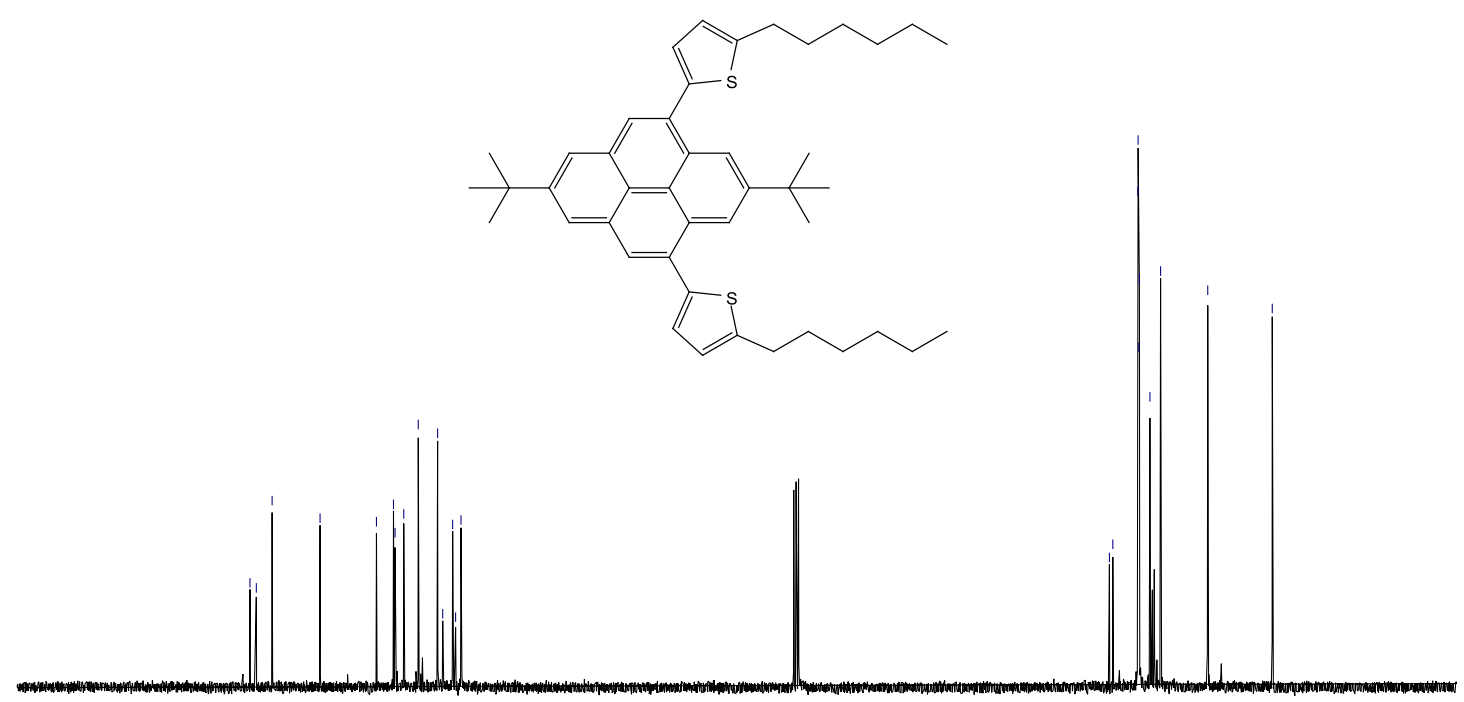

180

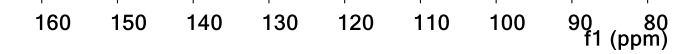

Figure S13. ${ }^{13} \mathrm{C}$ NMR spectrum for compound 7 in $\mathrm{CDCl}_{3}$ at $298 \mathrm{~K}$. 


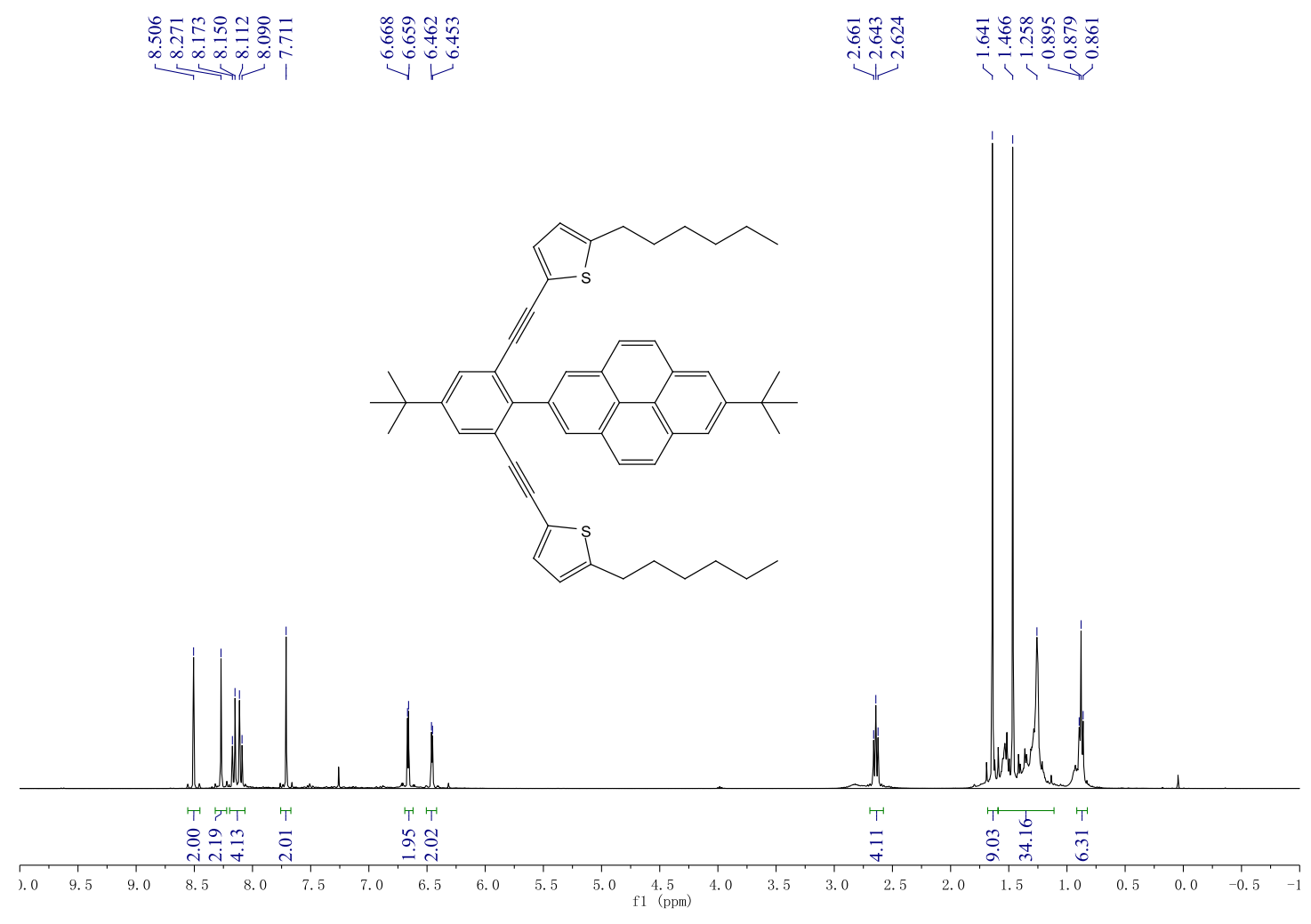

Figure S14. ${ }^{1} \mathrm{H}$ NMR spectrum for compound 9 in $\mathrm{CDCl}_{3}$ at $298 \mathrm{~K}$.

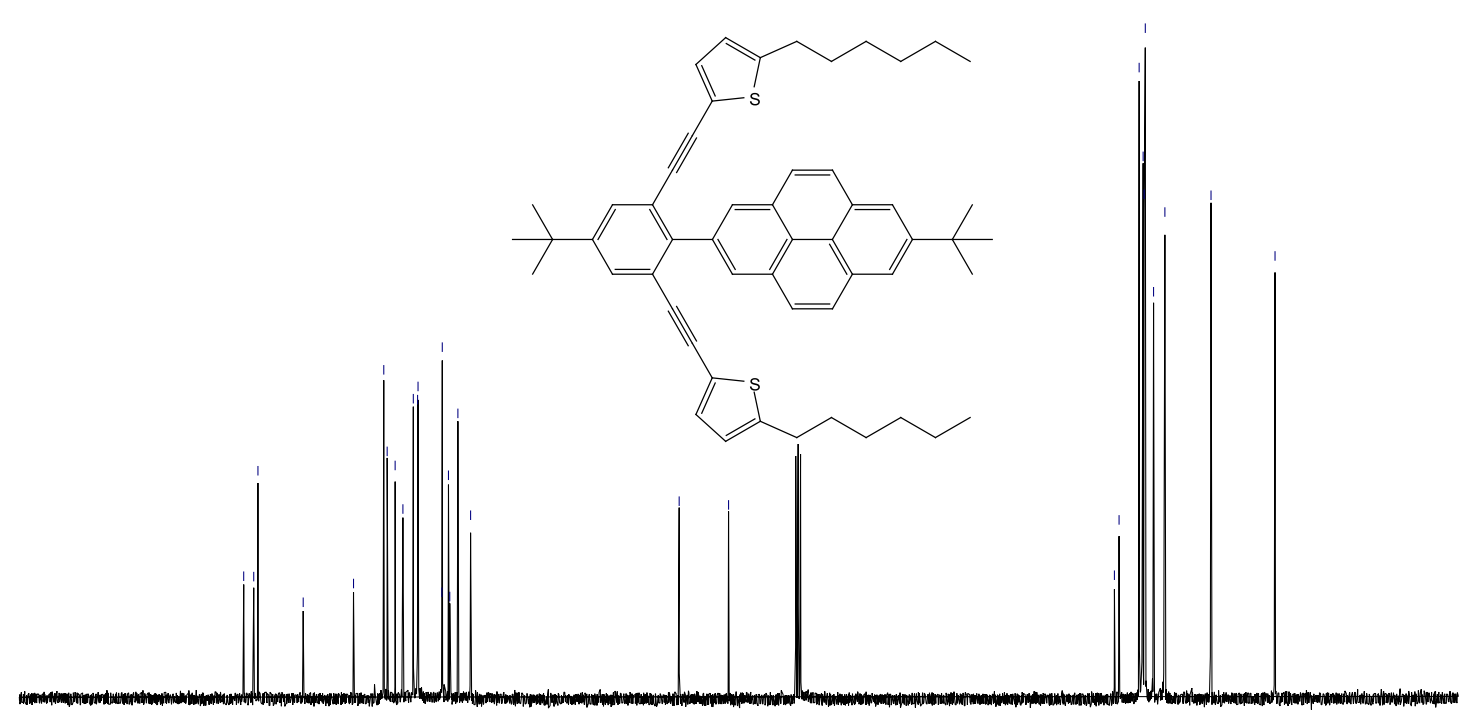

Figure S15. ${ }^{13} \mathrm{C}$ NMR spectrum for compound 9 in $\mathrm{CDCl}_{3}$ at $298 \mathrm{~K}$. 


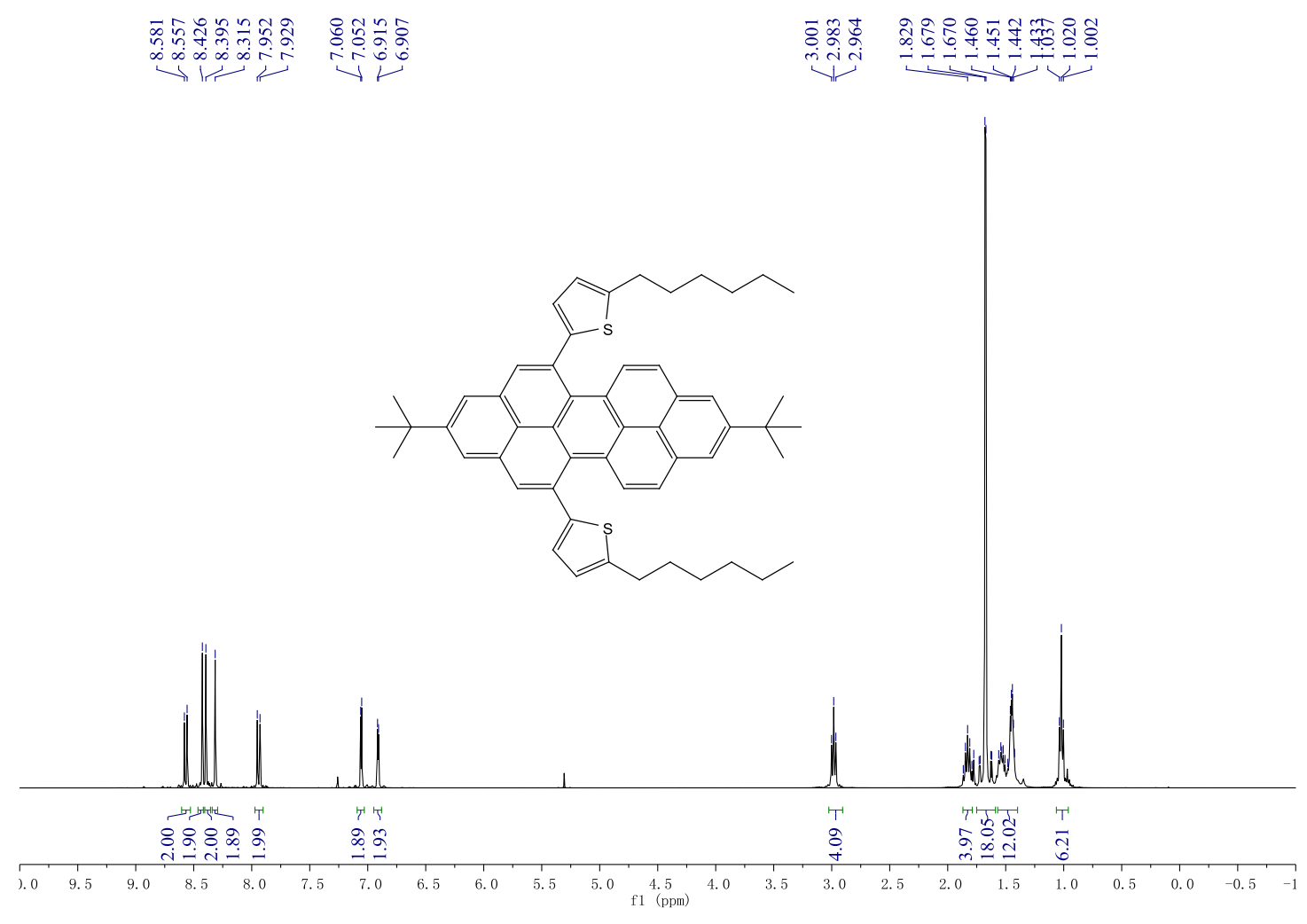

Figure S16. ${ }^{1} \mathrm{H}$ NMR spectrum for compound $\mathbf{1 0}$ in $\mathrm{CDCl}_{3}$ at $298 \mathrm{~K}$.
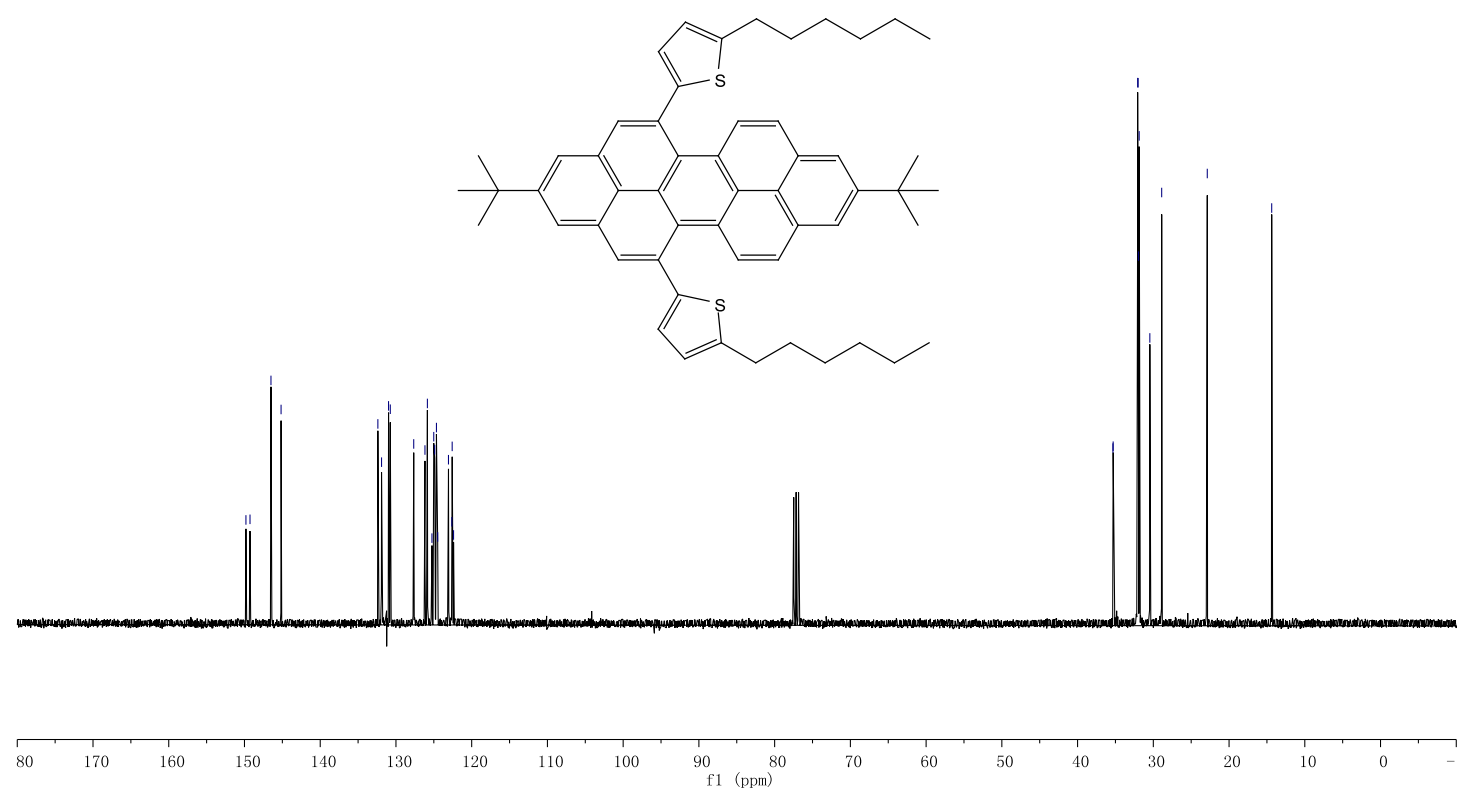

Figure S17. ${ }^{13} \mathrm{C}$ NMR spectrum for compound $\mathbf{1 0}$ in $\mathrm{CDCl}_{3}$ at $298 \mathrm{~K}$. 


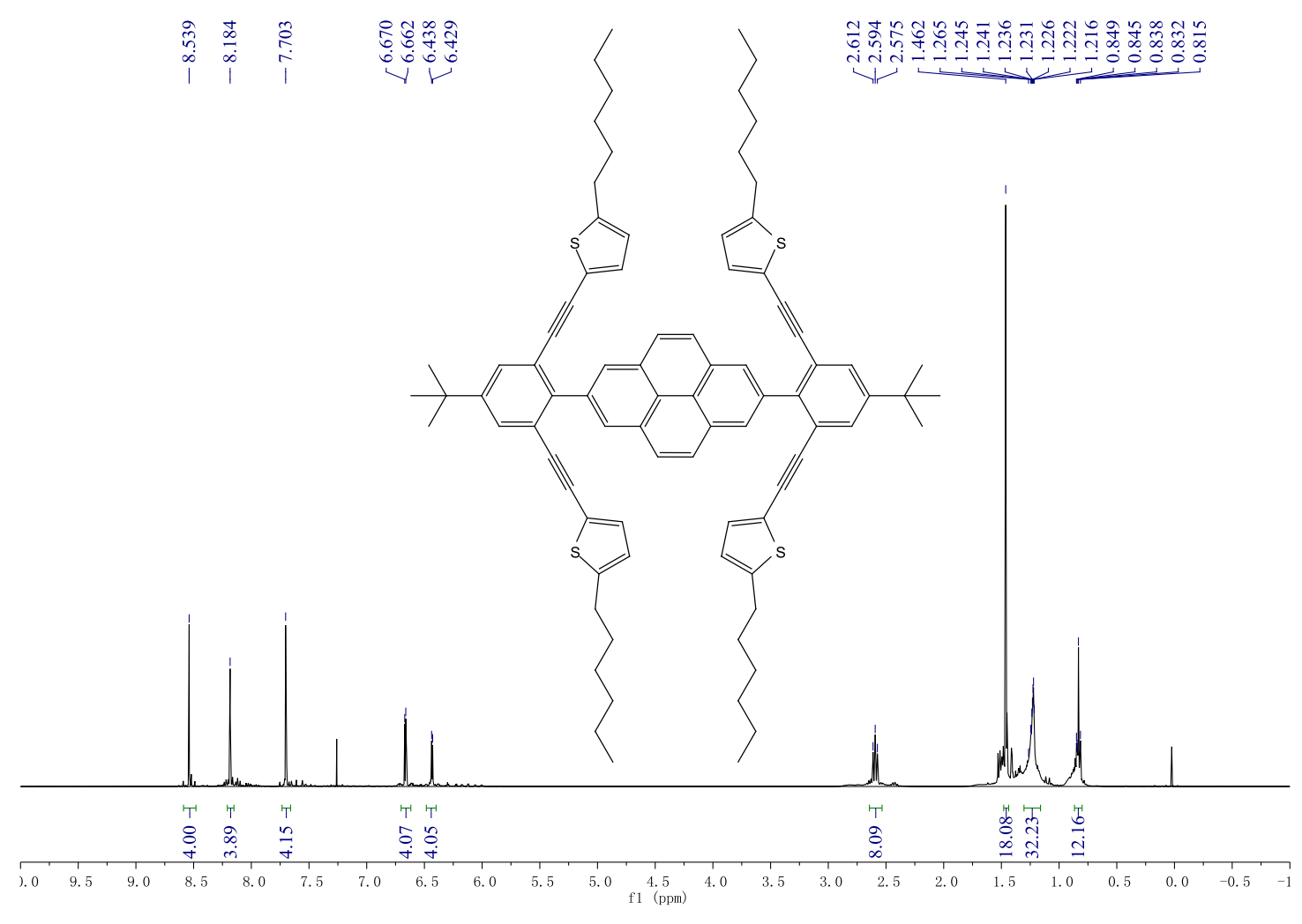

Figure S18. ${ }^{1} \mathrm{H}$ NMR spectrum for compound 12 in $\mathrm{CDCl}_{3}$ at $298 \mathrm{~K}$.
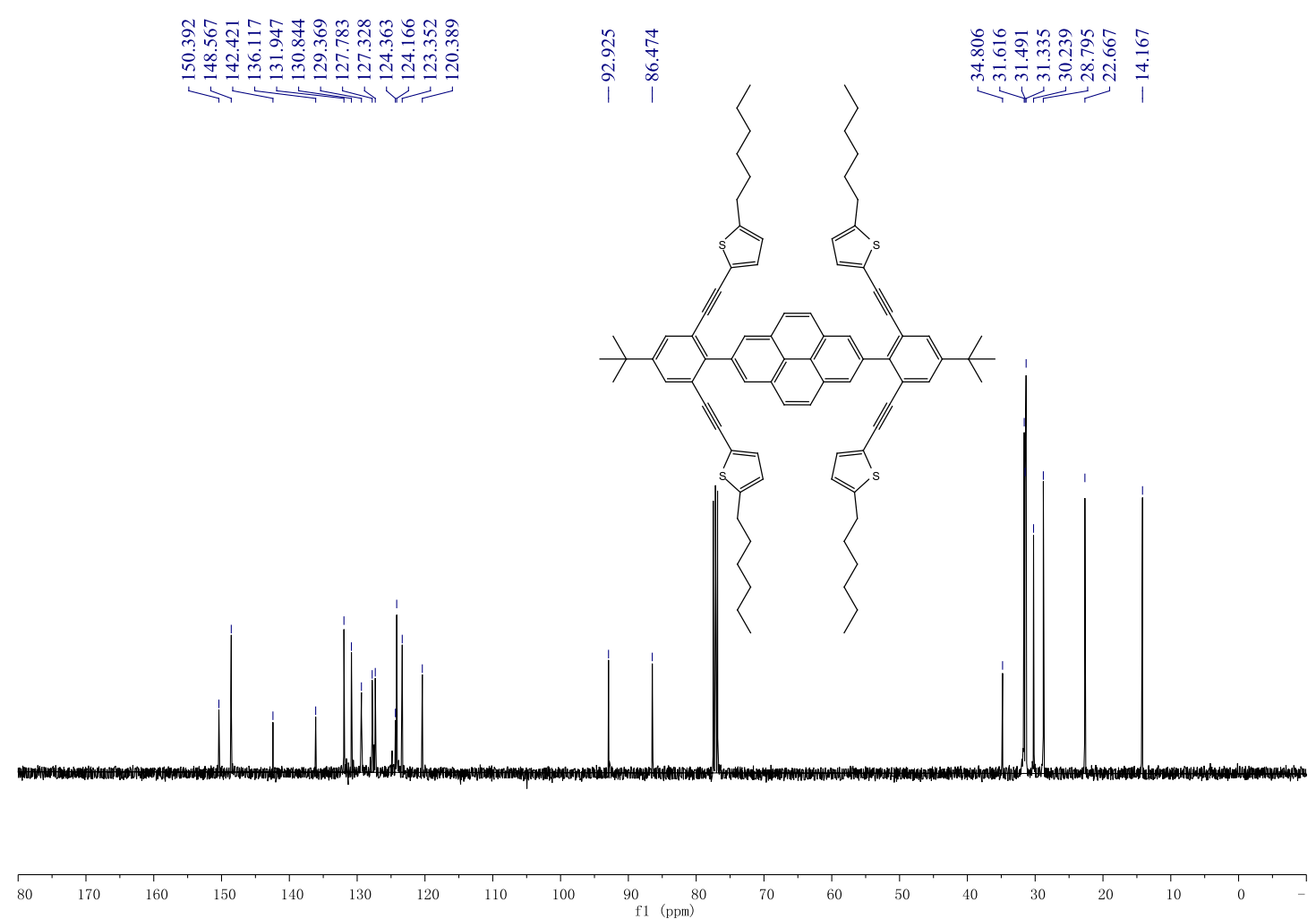

Figure S19. ${ }^{13} \mathrm{C}$ NMR spectrum for compound 12 in $\mathrm{CDCl}_{3}$ at $298 \mathrm{~K}$. 


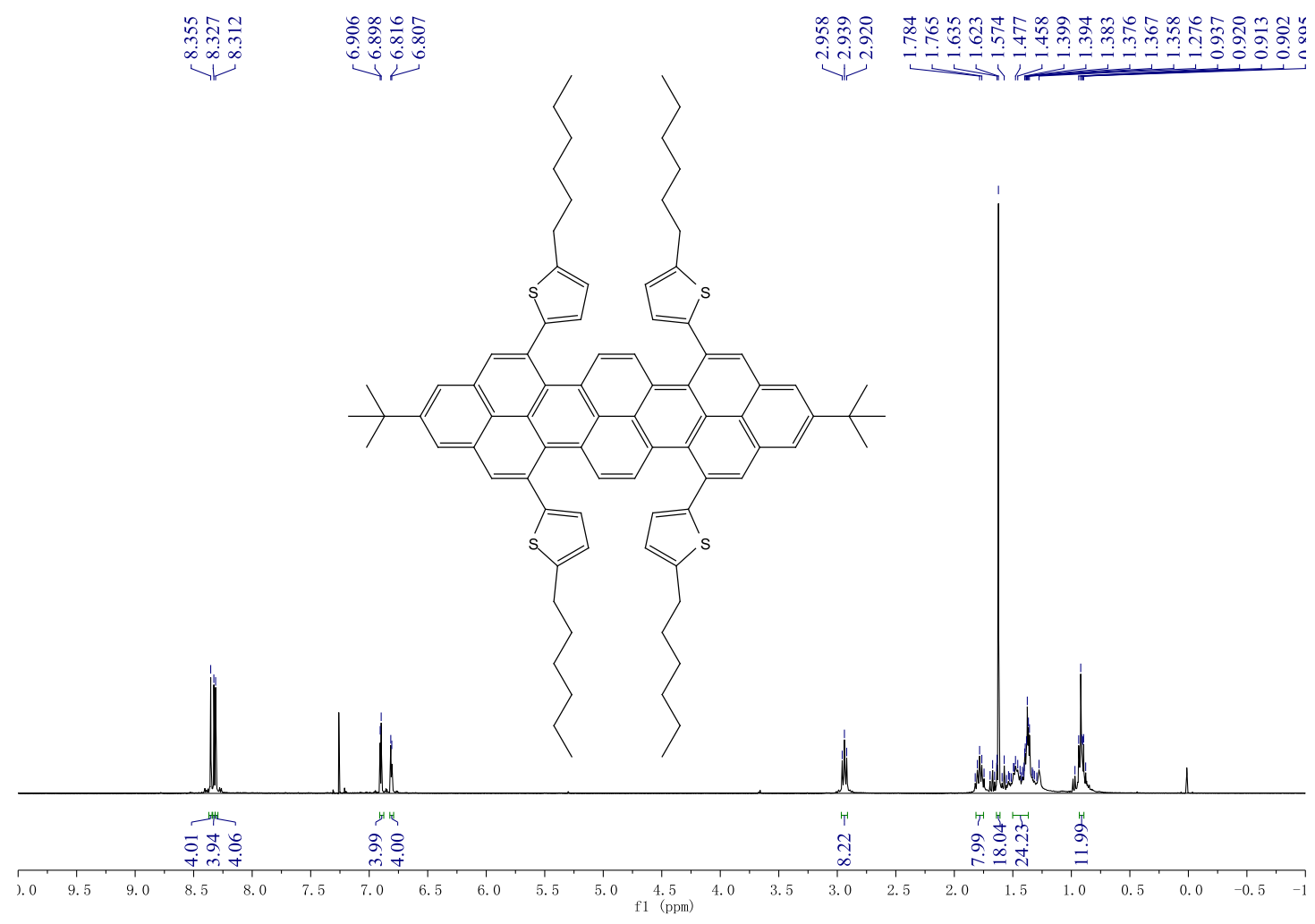

Figure S20. ${ }^{1} \mathrm{H}$ NMR spectrum for compound 13 in $\mathrm{CDCl}_{3}$ at $298 \mathrm{~K}$.
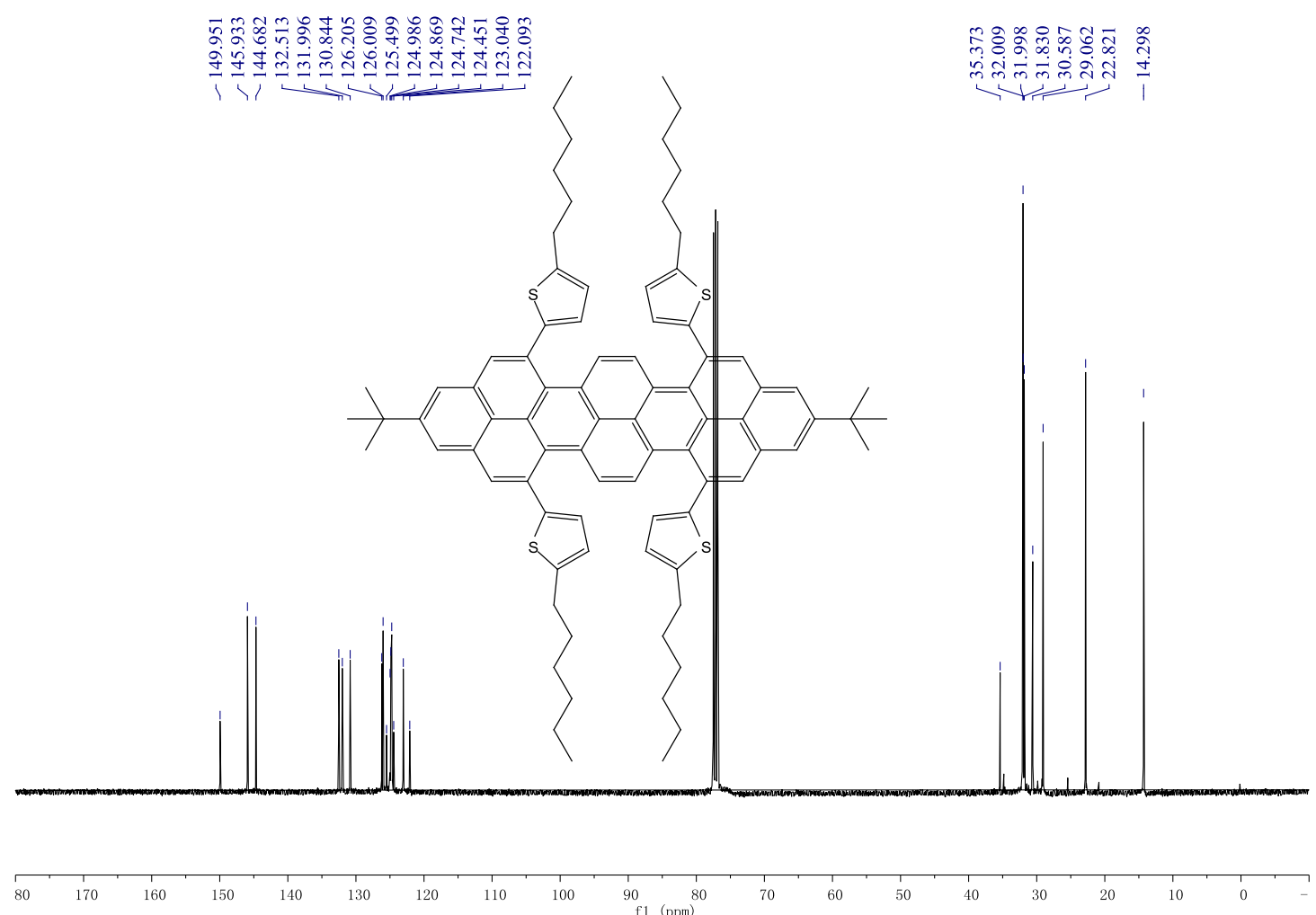

Figure S21. ${ }^{13} \mathrm{C}$ NMR spectrum for compound 13 in $\mathrm{CDCl}_{3}$ at $298 \mathrm{~K}$. 


\section{References:}

[1]. SMART: v.5.626, Bruker Molecular Analysis Research Tool. 2002.

[2].SAINTPlus: v.6.36a, Data Reduction and Correction Program, Bruker AXS: Madison, WI, 2001.

[3].SADABS: v.2.01, an empirical absorption correction program. 2001.

[4]. SHELXTL: v.6.10, Structure Determination Software Suite, Sheldrick, G.M. 2001.

[5].W. Yang, J. H. S. K. Monteiro, A. de Bettencourt-Dias, V. J. Catalano, W. A. Chalifoux, Angew. Chem. Int. Ed. 2016, 55, 10427-10430.

[6]. B. Shaik, J. H. Park, T. K. An, Y. R. Noh, S. B. Yoon, C. E. Park,Y. J. Yoon, Y.H. Kim, S.-G. Lee. Tetrahedron 2013, 69, 8191-8198.

[7].W. H. Melhuish, J. Phys. Chem. 1961, 65, 229-235.

[8]. M. Brouwer Albert, Pure Appl. Chem. 2011, 83, 2213-2228.

[9]. K. Nakamaru, Bull. Chem. Soc. Jpn. 1982, 55, 2697-2705. 